Nouvelles perspectives en sciences sociales

\title{
La place de la formation dans les stratégies d'adaptation industrielle : entre mondialisation et territoire
}

\section{Simon Laflamme et Alain Taché}

Volume 1, numéro 1, mars 2005

URI : https://id.erudit.org/iderudit/602448ar

DOI : https://doi.org/10.7202/602448ar

Aller au sommaire du numéro

Éditeur(s)

Prise de parole

ISSN

1712-8307 (imprimé)

1918-7475 (numérique)

Découvrir la revue

Citer cet article

Laflamme, S. \& Taché, A. (2005). La place de la formation dans les stratégies d'adaptation industrielle : entre mondialisation et territoire. Nouvelles

perspectives en sciences sociales, 1(1), 109-160. https://doi.org/10.7202/602448ar 
À cause d'une erreur de logiciel qui s'est glissée dans l'article de Simon Laflamme et Alain Taché, certains chiffres dans les tableaux 1 à 5 ont été corrompus. Prière de leur substituer les chiffres ci-dessous.

Tableau 1

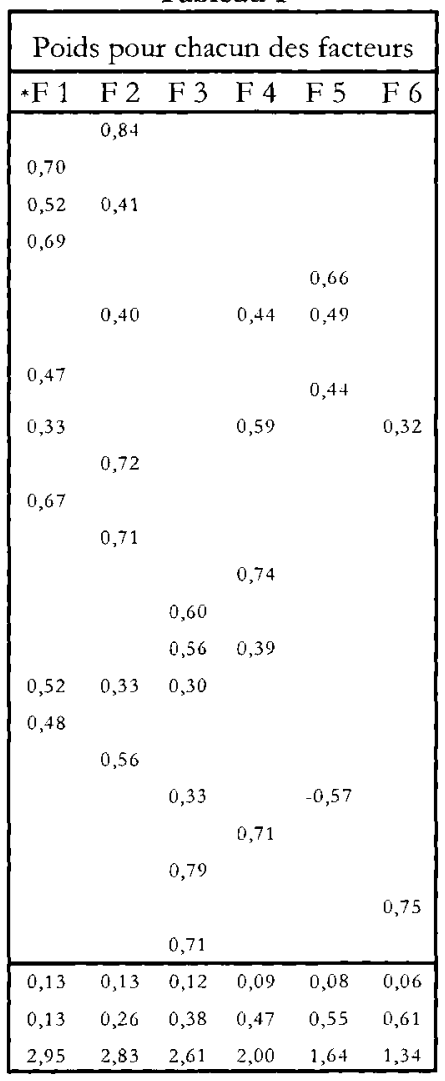

$* F=$ facteur
Tableau 2

\begin{tabular}{|c|c|}
\hline$\beta$ & $\mathrm{R}^{2}$ \\
\hline $\begin{array}{l}0,17 \\
0,18 \\
0,17\end{array}$ & 0,11 \\
\hline - & - \\
\hline 0,21 & 0,04 \\
\hline $\begin{array}{r}0,28 \\
0,25 \\
-0,22\end{array}$ & 0,13 \\
\hline $\begin{array}{r}0,41 \\
0,19 \\
0,21 \\
-0,20\end{array}$ & 0,27 \\
\hline 0,20 & 0,04 \\
\hline $\begin{array}{r}0,42 \\
-0,38 \\
0,19 \\
0,18 \\
0,16\end{array}$ & 0,30 \\
\hline $\begin{array}{r}0,33 \\
-0,37 \\
0,20 \\
0,21 \\
0,18\end{array}$ & 0,24 \\
\hline $\begin{array}{r}0,41 \\
0,26 \\
-0,21 \\
0,13 \\
0,20 \\
0,15\end{array}$ & 0,38 \\
\hline $\begin{array}{l}0,31 \\
0,25\end{array}$ & 0,19 \\
\hline $\begin{array}{r}0,24 \\
0,29 \\
-0,26\end{array}$ & 0,16 \\
\hline 0,25 & 0,13 \\
\hline 0,18 & 0,03 \\
\hline $\begin{array}{r}0,25 \\
-0,19\end{array}$ & 0,08 \\
\hline $\begin{array}{r}0,30 \\
-0,19\end{array}$ & 0,11 \\
\hline $\begin{array}{l}0,24 \\
0,18\end{array}$ & 0,09 \\
\hline $\begin{array}{r}-0,32 \\
0,22 \\
0,19\end{array}$ & 0,15 \\
\hline $\begin{array}{r}0,30 \\
-0,24 \\
0,18\end{array}$ & 0.12 \\
\hline $\begin{array}{r}0,23 \\
-0,23 \\
0,21\end{array}$ & 0.13 \\
\hline 0,19 & 0,04 \\
\hline
\end{tabular}

Tableau 3

\begin{tabular}{|c|c|c|c|c|c|}
\hline \multicolumn{2}{|c|}{ Tableau 3} & \multicolumn{2}{|c|}{ Tableau 4} & \multicolumn{2}{|c|}{ Tableau 5} \\
\hline$\beta$ & $\mathrm{R}^{2}$ & & $\mathrm{R}^{2}$ & $\beta$ & $\mathrm{R}^{2}$ \\
\hline 0,24 & 0,06 & 0,21 & 0,07 & 0,24 & 0,14 \\
\hline 0.31 & 0,20 & 0,20 & & 0,20 & \\
\hline 0,24 & & 0,26 & 0,07 & $0 \times 0$ & 0 \\
\hline 0,27 & 0,07 & 0,17 & 0,03 & $\begin{array}{r}-0,38 \\
0,24\end{array}$ & 0,10 \\
\hline 0,32 & 0,22 & 0,31 & 0,16 & 0,20 & \\
\hline 0,24 & & 0,19 & & $(0,19$ & 0,04 \\
\hline 0,35 & 0,12 & 0,36 & 0,27 & & \\
\hline 0,28 & 0,12 & $\begin{array}{l}0,40 \\
0 ? 7\end{array}$ & & & \\
\hline 0,19 & & $-0,20$ & & & \\
\hline 0,22 & 0,14 & 0,25 & & & \\
\hline 0,20 & & $-0,17$ & & $-(1,24$ & 0,16 \\
\hline 0,16 & & 023 & 0,11 & 0,25 & \\
\hline 0,22 & 0,12 & 0,21 & & 0,19 & \\
\hline 0,20 & & 0,30 & 0,27 & 020 & 033 \\
\hline 0,43 & 0,26 & 0,27 & & $-0,29$ & \\
\hline 0,28 & & 0,18 & & 0,26 & \\
\hline$-0,22$ & & 0,32 & 0,30 & $-0,24$ & \\
\hline 0,30 & 0,09 & 0,30 & & 0,20 & \\
\hline 0,32 & 0,17 & $\begin{array}{r}-0,24 \\
0.19\end{array}$ & & 0,24 & 0,06 \\
\hline 0,22 & & $0 ح$ & & & \\
\hline 0,27 & 0,15 & $\begin{array}{l}0,26 \\
0,19\end{array}$ & 0,22 & & \\
\hline 0,23 & & 0,18 & & & \\
\hline 0,21 & 0,09 & 0,36 & 0,26 & & \\
\hline 0,17 & & $\begin{array}{r}0,29 \\
0,17\end{array}$ & & & \\
\hline 024 & 000 & 0,25 & 0,18 & & \\
\hline 0,16 & & 0,18 & & & \\
\hline 0,30 & 0,09 & 010 & 000 & & \\
\hline 0,30 & 0,17 & 0,18 & & & \\
\hline .020 & & 026 & 016 & & \\
\hline 0,19 & & 0,26 & 0,10 & & \\
\hline 0,31 & 0,10 & $-0,25$ & & & \\
\hline
\end{tabular}




\section{La place de la formation dans les stratégies d'adaptation industrielle : entre mondialisation et territoire}

\author{
Simon Laflamme \\ Département de Sociologie \\ Université Laurentienne \\ Sudbury, Ontario, Canada
}

\author{
Alain Taché \\ CIRESS et CRIDF \\ Université des Sciences Sociales \\ Toulouse, France
}

\section{Introduction}

Internationalisation, européanisation, délocalisation, désindustrialisation... autant de processus, centraux pour l'avenir de nos sociétés, qui font l'objet de nombreuses recherches en sciences sociales. Menaces pour les uns, opportunités pour les autres, synonymes de réification morbide pour certains ou d'émergence de nouveaux modèles pour d'autres, ces processus ne laissent pas indifférents tant ils sont au cœur des relations entre le global et le local, entre l'Europe et ses membres, entre les États et leurs régions, tant ils sont, finalement, au cœur de la construction socio-économique et de l'activité sociétale.

Notre projet est ici de rendre compte de la conjonction paradoxale des hypothèses de la mondialisation et de la postmodemité dans la compréhension des stratégies d'adaptation des entreprises qui, face aux mutations internes et externes, ont recours à la formation professionnelle comme ressource à leur processus d'adaptation. Pour ce faire, nous argumenterons, tout d'abord, le constat d'un glissement sémantique - de la mutation à l'adaptation - sans doute révélateur de changements de conceptions, d'une 
transformation de la représentation que les chercheurs se font du phénomène. Nous préciserons, ensuite, le questionnement, les hypothèses et les méthodes qui ont guidé notre projet; approche que l'on peut qualifier de systémique dans le sens d'une façon d'aborder les données qui cherche à relier des variables pour rendre compte de combinaisons au-delà de corrélations strictes.

En France, la Délégation à l'Aménagement du Territoire et à l'Action Régionale (DATAR), depuis plusieurs années, propose, plutôt que d'aborder ces phénomènes en terme de désindustrialisation, de parler de mutations industrielles. Le rapport de février 2004 - L a France, puissance industrielle - fait une synthèse du point de vue de la France dans le concert européen.

L'argument repose sur une approche globale et décloisonnée dans le sens où, par exemple, la DATAR montre que les emplois de l'intérim sont classifiés dans le secteur des services alors que 300000 intérimaires travaillent dans l'industrie. Dans le même ordre d'idée, la DATAR relève que les statistiques sur le secteur industriel ne prennent pas en compte les mouvements d'externalisation de certaines productions, jusqu'alors comptabilisés dans l'industrie, qui glissent vers d'autres secteurs, notamment celui des services. Les grilles de lecture, telles que par exemple les nomenclatures de métiers, relevant d'approches exclusives et cloisonnées, sont mises en question et ce qui apparaissait comme un processus de désindustrialisation dans une approche sectorielle peut se représenter comme un processus de mutation industrielle dans une approche intersectorielle et globale.

La notion même de mutation industrielle s'en trouve affectée. Longtemps entendue dans une acception " industrialiste », la mutation industrielle est définie aujourd'hui de manière large et ouverte. Il s'agit, bien sûr, de mutations technologiques, mais on a également affaire à des transformations socio-organisationnelles internes à l'entreprise ou à des changements socio-organisationnels entre l'entreprise et son environnement. Il peut s'agir aussi de changements profonds qui ont cours dans certains secteurs professionnels qui ne sont pas exclusivement industriels mais qui appartiennent à une problématique d'adaptations profondes dans le système des relations de travail (organisation du travail, exigences des relations entre donneurs d'ordres et sous-traitants, nouvelles compétences attendues, signature d'accord de branche, etc.). Tout se passe comme si la mutation industrielle, en tant que processus de transformation socio-économique, reprenait sa place au sein d'un processus plus large qui est celui de l'adaptation industrielle et cédait la place à cette notion d'adaptation qui est aujourd'hui plus volontiers utilisée pour se représenter ces phénomènes de transformation. 
Pour la DATAR, la question est alors celle de l'accompagnement de ce processus. Soutenue en cela par le Fonds Social Européen (FSE), l'accompagnement de l'adaptation industrielle est au coeur des politiques actives de lutte contre le chômage depuis de nombreuses années en France. La DATAR, aujourd'hui, renforce cette position et plaide pour un renforcement par les territoires d'une politique industrielle.

Dans la panoplie d'instruments pour une ré-ingénierie de la politique industrielle et pour accompagner les entreprises dans ce processus de transformation, la formation professionnelle occupe une place privilégiée aux côtés d'autres leviers tels que, par exemple, les réductions du coût du travail. Elle apparaît comme une ressource mobilisable dans le processus d'adaptation industrielle, voire de transformation sociétale. Au-delà d'une conjoncture économique fluctuante, les entreprises, pour construire leur développement, doivent tenir compte d'une multitude de facteurs, notamment, le renouvellement à venir des effectifs liés au vieillissement de la population active, la création de nouveaux produits, l'évolution des organisations ou encore le progrès technologique. Autant de facteurs qui nourrissent la dynamique de l'adaptation industrielle et par rapport auxquels la formation professionnelle apparait comme le trait d'union avec les actions à mener. Aujourd'hui, il n'est pas une proposition, pas un projet en matière de politique industrielle qui ne fasse référence à la formation professionnelle des individus. Concomitamment, ces individus sont de plus en plus responsabilisés quant à leur situation professionnelle. Pour le chercheur d'emploi, la formation est présentée comme un outil d'amélioration de sa qualification et comme le sésame du retour au travail; pour le travailleur en poste, la formation représente la possibilité d'actualiser ses compétences pour prévenir tout risque d'exclusion du marché du travail.

C'est dans ce contexte de transformation tous azimuts et dans cet esprit que l'Objectif IV du FSE soutient les projets d'entreprises dont la formation professionnelle des salariés est un axe d'action prioritaire. Le FSE intervient comme cofinanceur des projets et prend place auprès de financements privés, issus de l'entreprise, et / ou publics (provenant de l'État, des collectivités territoriales) $)^{1}$. Il souhaite ainsi « donner une impulsion considérable au processus d'adaptation des salariés aux mutations industrielles en France $»^{2}$. Les projets cofinancés doivent répondre à des critères précis d'éligibilité. Les

\footnotetext{
${ }^{1}$ Les collectivités territoriales sont, en France, la région, le département, la commune ou la communauté de communes.

${ }^{2}$ Document Unique de Programmation (DOCUP) Objectif IV, "Favoriser l'adaptation des travailleurs aux mutations industrielles et à l'évolution des systèmes de production », publié par le Ministère de l'emploi et de la solidarité.
} 
projets soutenus par des petites et moyennes entreprises ${ }^{3}$ (PME) sont en principe prioritaires. Les projets doivent bien évidemment avoir un lien avec les mutations industrielles et les transformations des systèmes de production. Ils doivent, en outre, s'inscrire dans une démarche anticipative, s'adresser à des travailleurs menacés par les transformations précitées, être transversaux (intersectoriels) ou encore avoir un caractère innovant. Les projets sont déposés auprès de chaque Direction Régionale du Travail, de l'Emploi et de la Formation Professionnelle (DRTEFP), instance déconcentrée de l'État en Région. Ils sont instruits par les membres de la Cellule FSE, laquelle constitue l'interlocuteur privilégié pour diffuser l'information, pour accompagner techniquement les porteurs de projets dans la mise en œuvre de leurs actions et pour évaluer l'impact du cofinancement.

Au terme de la demière programmation (1994-2001), la DRTEFP de Midi-Pyrénées a souhaité passer commande auprès d'une équipe pluridisciplinaire de chercheurs d'une évaluation finale de l'impact des cofinancements du FSE-Objectif IV dans les processus d'adaptation industrielle des entreprises de son territoire. ${ }^{4}$ Notre contribution prend appui sur une partie des données recueillies (interviews, questionnaires, monographies) dans ce cadre et se focalise sur quelques questions de recherche qui, tout en étant articulées à l'évaluation, en sont indépendantes. Il s'agit, ici, de reprendre quelques-unes des questions qui sous-tendent l'évaluation de l'apport du FSE dans les processus d'adaptation industrielle des entreprises du territoire de MidiPyrénćes, dans une visée illustrative de différents niveaux d'hypothèses entremêlées. Quels sont ces niveaux?

Les réflexions de la DATAR sont significatives d'un mouvement ample de réflexions et d'un questionnement théorico-pratique profond sur les processus à l'œuvre dans les transformations sociétales. Comme dans bien d'autres domaines, il n'est pas innocent que, au fil des programmations, le vocabulaire construit pour désigner les phénomènes socio-économiques évolue. On est passé, dans les textes de référence, de la mutation industrielle à l'adaptation industrielle. Comment comprendre ce passage? Comment se représenter ce glissement sémantique? De quelles dimensions théoricopratique est-il porteur?

Il est évident que la référence implicite aux termes de mutations et d'adaptation est le corpus théorique de la biologie. Le monde de l'industrie, comme celui d'autres sphères de l'activité humaine, a imaginé, par analogie à

\footnotetext{
${ }^{3}$ En France, moins de 200 salariés.

${ }^{4}$ Le Centre de Recherche et d'Information sur le Droit à la Formation de l'Université des Sciences Sociales de Toulouse a remis un rapport d'évaluation aux autorités compétentes en mars 2001.
} 
la biologie, des modèles théoriques permettant de se représenter les transformations qui l'affectent. Un des premiers chercheurs qui ont conceptualisé l'adaptation en biologie est sans doute Lamarck (1968) qui, s'opposant à l'idée de fixité des espèces, a le pressentiment de l'origine à la fois endogène et exogène de l'adaptation. Pour lui, l'adaptation, qui est le moteur de la transformation des espèces, est gouvemée par la conjonction de forces organisatrices et de forces désorganisatrices. Plus tard, Danwin (1983 [1859]) propose une autre conceptualisation et les mécanismes de la "sélection naturelle " repris de sa théorie ont inspiré largement certains modèles économiques (Tort, 1996; Nelson et Winter, 1982; Williamson 1974, 1975, 1976 ). Dans cette conceptualisation, l'adaptation industrielle ne peut se comprendre sans la sélection naturelle qui est le moteur de la transformation industrielle. L'adaptation n'est pas une propriété des entreprises mais la résultante d'un mécanisme discriminatoire. La sélection naturelle ne décrit pas les processus qui permettent aux entreprises d'être adaptées mais montre, dans la lutte pour l'existence, la nécessaire compétition entre entreprises. Cette compétition aboutit à préserver les entreprises les plus aptes à exister dans les conditions imposées par le marché et par la lutte entre congénères. L'adaptation par sélection naturelle est le produit d'une coïncidence entre les caractéristiques que l'entreprise avait déjà avant d'être sélectionnée, d'une part, et, d'autre part, les caractéristiques du milieu économique à ce moment. Les conceptions mutationnistes de la transformation des espèces ont, elles aussi, permis, toujours par analogie, d'enrichir les modèles de l'analyse de la transformation des sphères d'activités industrielles. Tout comme en biologie pour les êtres vivants, la mutation est une des modalités d'adaptation des entreprises, mais c'est seulement une des modalitẻs possibles dans la variété des scénarios susceptibles d'être mis en œuvre. La mutation industrielle peut être considérée comme une modification brusque à l'origine d'une nouvelle organisation (apparition de nouvelles molécules, déclenchement de nouveaux processus...); or, force est de constater qu'il s'agit là d'une représentation pauvre et réductrice des transformations possibles d'une entreprise et, partant, d'un secteur industriel.

Aujourd'hui, le monde de l'industrie, s'il est autonome dans sa conceptualisation (Alcaras et Lacroux, 2001; Zarifian et Palloix, 1988; Jacot, 1990), puise toujours dans ses racines biologiques quelques analogies, quelques images, quelque vocabulaire. L'adaptation fait partie de ces mots qui traversent les disciplines et les champs de l'activité humaine (Boyer et Freyssenet, 1995). Le choix de tel vocable ou de tel autre n'est pas innocent; il est, selon nous, en référence étroite à une transformation du point de vue duquel les chercheurs, notamment ceux de la DATAR, se placent pour repérer leur objet. Il serait 
d'ailleurs intéressant de vérifier cette hypothèse que, au travers de l'adoption d'un nouveau vocabulaire, nous assistons au changement de regard porté sur les phénomènes de transformation industrielle et, en conséquence, à la transformation de la conceptualisation des phénomènes. Mais notre projet ne se situe pas à ce niveau. Admettons simplement que, face à «la mutation », «l'adaptation» traduit sans doute le souhait des chercheurs de la DATAR de se représenter le phénomène de transformation industrielle de manière plus large, moins catégorielle, plus ouverte, moins cloisonnée, plus systémique voire plus complexe. En fait, il nous semble que la postmodemité a mis en évidence certaines caractéristiques de l'action sociale, a précisé certains termes de l'environnement socio-économique de telle manière qu'une approche plus nuancée de l'objet s'est imposée. La mondialisation a rendu plus incisif le libéralisme économique, elle a inscrit la concurrence dans un mode continu; ce faisant, elle a obligé les acteurs économiques à intervenir sur eux-mêmes et sur le milieu de façon constante. En accroissant la concurrence, elle a aussi diversifié et étendu la dimension économique de la socialité; cette évolution a favorisé aussi bien la diversification des interventions socio-économiques que leur uniformisation, car elle a pluralisé aussi bien les zones d'interventions que les actions individuelles en même temps qu'elle leur a imposé un cadre globalisant. Cette homogénéisation et, paradoxalement, cette différenciation, qui sont inhérentes à la socialité postmoderne en tant qu'elle participe de la mondialisation (Laflamme et Reguigui, 2003), ont contribué à mettre en lumière les dimensions autres que rationnelles dans la psyché des acteurs sociaux; l'idée d'un acteur intégralement rationnel, même au plan économique, ne peut plus être soutenue; si cet acteur est capable de décisions intentionnées, et donc rationnelles, il est tout aussi en mesure de mettre en œuvre des positions émotives, voire irrationnelles. Les représentations de l'acteur économique sont complexes: mouvantes et récurrentes, rationnelles et non rationnelles, ambiguës aussi bien que nettes; elles sont contraintes à une vision du monde qui les inscrit dans la continuité et, donc, dans la transformation. La postmodemité impose cette évidence. Et c'est sans doute pour cette raison que le vocabulaire des intervenants politico-économiques s'est déplacé d'une logique de la mutation vers une logique de l'adaptation.

Si cela est vrai, nous devrions trouver la trace de cette complexité dans les représentations des acteurs qui portent l'adaptation de l'entreprise? S’il est plus satisfaisant de se représenter le processus d'adaptation de manière systémique, ne faut-il pas repérer auprès des porteurs de projets d'adaptation une représentation que l'on pourra qualifier de systémique? Là est notre hypothèse, dans cette idée que, si les chercheurs de la DATAR ont raison et qu'il est plus sensé de changer de vocabulaire, alors il faut être en mesure de 
développer une modélisation qui donne à voir une représentation sociale du phénomène d'adaptation industrielle qui traduise cette complexité.

La question se pose alors de l'étayage théorique d'une telle définition de l'adaptation. Comment, en effet, caractériser l'adaptation industrielle qui serait systémique, voire complexe? Un des points communs aux modélisations systémiques de Simon, Alcaras ou encore de Le Moigne est sans doute la possibilité de se représenter l'adaptation de l'entreprise en relation avec l'existence d'un système de traitement de l'information et d'un système de décision.

L'idée est ici que l'adaptativité de l'entreprise, en tant qu'organisation, repose notamment sur sa capacité à traiter l'information, d'où qu'elle vienne. Ces informations, de différents ordres et de différents niveaux, peuvent se repérer au travers de deux grandes catégories que sont l'environnement et les finalités. Les finalités sont plus ou moins changeantes et l'environnement est également plus ou moins fluctuant. En conséquence, les informations sont aussi susceptibles d'incertitudes, d'ambiguités, de polysémies. Quel que soit le point de vue adopté, ce système permet, dans le cadre du processus d'adaptation, le recueil, la caractérisation, la catégorisation, l'agencement, le rapprochement, la signification; bref, il permet de traiter les indices et les symboles qui proviennent à la fois de l'environnement et de l'entreprise ellemême. Il occupe une position stratégique importante puisque c'est lui qui permet à tout moment de transmettre à l'entreprise les fluctuations issues de l'environnement ou de l'entreprise elle-même. De par son organisation et de manière paradoxale, le système, tout à la fois, traite de l'information et masque de nombreux renseignements, voire désinforme.

On peut alors dire que les informations, et donc les problèmes à résoudre pour l'entreprise, ne sont en aucun cas traitées comme des données strictement objectives. Elles passent, en effet, au crible d'un réseau cognitif, ou plutôt « émorationnel » (Laflamme, 1995), associant des schèmes de tous ordres et de tous niveaux qui, construits antérieurement, sont plus ou moins formalisés et qui, finalement, sont mobilisés par l'entreprise en fonction de son projet, de ses finalités. Le réseau lui-même se construit au fur et à mesure de l'évolution de la compréhension de la situation. Nous sommes loin d'une conception rationnelle du traitement de l'information et nous nous rapprochons davantage d'une conception procédurale qui « met l'accent sur les processus adaptatifs de recherche de solutions localement et momentanément satisfaisantes, tirant parti notamment des facultés d'apprentissage et d'invention » (Le Moigne, 1995).

L'entreprise, comme l'a largement argumentée Simon (1983, 1991), à l'instar des sujets qui la composent, ne prend d'ailleurs des décisions que 
satisfaisantes et non pas optimales. Non seulement les filtres internalisés conditionnent le processus de traitement de l'information mais, de plus, l'entreprise ne peut, de manière exhaustive, recueillir toute l'information. Du point de vue de l'information recueillie et traitée, mais aussi du point de vue d'un schéma de la prise de décision, les décisions ne peuvent être alors que satisfaisantes, dans les circonstances.

De manière schématique, pour prendre une décision, l'entreprise cherche à se représenter la situation dans laquelle elle se trouve. Elle procède en quelque sorte à un état des lieux de la situation en relation avec son projet plus ou moins construit et susceptible d'aménagements. Quel estl'environnement dans lequel elle évolue? Quels sont ses objectifs, ses projets? Quelles sont les actions déjà développées et quels sont les résultats? Quelles sont les ressources dont elle dispose? Quels sont les décalages entre ce qu'elle souhaitait et ce qu'elle a réalisé? L'entreprise cherche par cet état des lieux, et au moment où il a lieu, à comparer ce à quoi elle veut parvenir avec ce à quoi elle est parvenue. Cette comparaison est l'une des premières étapes de tout processus de décision dont Simon (1983) rend compte, par le modèle Intelligence-Conception-Sélection (I.C.S.) ${ }^{5}$. Simon développe trois étapes caractéristiques que l'on peut repérer comme étant, d'abord, celle de la comprébension du problème, ensuite, celle de la conception et l'évaluation des solutions possibles à mettre en cuvre et, enfin, celle du moment de la sélection de l'option choisie qui peut, selon le cas, renvoyer à une réflexion supplémentaire, une recherche d'information complémentaire, une réactualisation des finalités ou un passage à l'action. Il s'agit donc de poser les problèmes ou plus exactement de les construire. La compréhension est le " processus par lequel le problème décisionnel est construit. Elle se développe par un exercice de mise en rapport symbolique, exprimant la qualité de l'adaptation téléologique du système complexe à chaque instant: qualité qui s'apprécie par le rapport entre la situation perçue et la situation projetée » (Le Moigne, 1995: 132). Ainsi, il ne s'agit pas de considérer que les problèmes sont déjà bien posés. C'est d'ailleurs toute la différence entre les activités décrites par Simon de problem finding et de problem solving que souligne Alcaras (1997) dans sa thèse quand il écrit: « Est-ce une banalité que de rappeler l'importance de cette phase préalable? On peut au moins admettre qu'on la néglige bien souvent... Certains décideurs s'égarent ainsi à cause d'une fâcheuse tendance à considérer que les problèmes sont définis une fois pour toutes et de manière stéréotypée, ce qui les conduit à privilégier en fait les problèmes qui sont structurés d'une manière qui leur permet d'utiliser les outils décisionnels formels qu'ils connaissent et utilisent habituellement. D'ailleurs, à l'école, apprend-on aux

${ }^{5}$ Herbert A. Simon, Administration et processus de décision, Paris, Economica, 1983. 
élèves à trouver, à poser et à formuler des problèmes, ou bien plutôt à résoudre des problèmes, certes compliqués, mais déjà bien posés? Cela revient à mettre la charrue avant les bouffs. les problèmes ne sont pas des données de la nature, ils restent très généralement à poser ». Il s'agit ensuite de construire des scénarios, des plans d'actions ou des stratégies possibles qui répondent aux décalages repérés lors de la phase de compréhension du problème. Le Moigne définit l'« ingenium $»^{6}$ comme la capacité qui permet cette construction de scénarios qui, une fois conçus, sont évalués au regard de différents critères préalablement construits en relation au projet et aux écarts constatés en début de processus de décision. La sélection de l'option choisie est, quant à elle, « le processus par lequel le système compare les évaluations des plans d'action (ou des propositions de solutions) élaborés lors du processus de conception » (Le Moigne, 1995: 133). Les acteurs concernés par la décision peuvent, après délibération, choisir d'agir, de s'informer (reconstruire le problème, concevoir d'autres possibilités) ou de modifier en tout ou en partie les finalités du système.

Soulignons ici avec Alcaras que l'étude attentive du modèle développé par Simon fait apparaître que " dans un processus où un individu cherche à adapter son comportement aux évolutions qu'il perçoit dans son environnement et conformément aux projets qu'il poursuit, les actions symboliques sont au moins aussi importantes que les actions physiques » (1997: 147). On remarquera en faveur de cet argument que les actions physiques ne représentent qu'une des quatre modalités de prise de décision. On notera surtout que les actions physiques sont elles-mêmes la résultante d'actions symboliques, c'est-à-dire de la manipulation de symboles.

Simon propose d'ailleurs de considérer le système de prise de décision comme système de traitement de symboles physiques. Il argumente l'idée que « les systèmes de symboles sont pratiquement les artefacts quintessenciels, parce que leur faculté d'adaptation à un milieu est leur seule raison d'être. Ce sont des systèmes finalisés de traitement d'information généralement au service des systèmes plus importants au sein desquels ils sont incorporés " (1991: 22).

Il met ainsi, comme le souligne Alcaras, l'accent sur «l'aspect intentionnel, finalisé de la représentation que l'on se fait du monde sur lequel on décide

\footnotetext{
${ }^{6}$ "L'Ingenium comme la faculté mentale qui permet de relier de manière rapide, appropriée et heureuse des choses séparées. Faculté synthétique, opposée à l'analyse stérile, elle permet l'invention et la création; elle est indispensable à la poésie, à l'invention technique des ingénieurs et à la découverte scientifique et philosophique ", Giambattista De Vico, La Méthode des études de notre temps, Grasset, Paris, 1981, cité par Le Moigne (1995: 133).
} 
d'agir: le symbole donne un sens à notre décision, à notre action et réciproquement » (1997: 148). De plus, « les systèmes de symboles sont appelés physiques pour rappeler au lecteur qu'ils existent comme instrument du monde réel, faits ici de verre et de métal (ordinateurs), ou là de chair et de sang (cerveaux)» (Simon, 1991: 23). Les symboles physiques sont réels dans le sens où ils conditionnent l'action réelle, c'est-à-dire les actions physiques.

Une telle définition n'est ni close, ni complète, ni définitive. Elle offre l'avantage de pointer que l'adaptation de l'entreprise est un processus global, endogène et permanent par lequel l'entreprise, en relation avec les sollicitations de son milieu interne et externe, modifie de manière délibérée (au travers d'un système d'information et de décision) et de façon plus ou moins importante, son niveau d'organisation. La nouvelle organisation obtenue n'est que le résultat effectif et satisfaisant du processus d'adaptation (Taché, 2003: 301). Certes, elle accorde beaucoup d'importance à la dimension rationnelle de la décision en insistant sur l’intention, sur le projet, sur la conscience, comme le veut l'appareil conceptuel des modèles rationalistes; mais elle n'exclut pas d'autres aspects de la psyché, même si elle a de la difficulté à les incorporer.

Si la réalité perçue par les chefs d'entreprises peut être lue au travers de cette perspective, alors nous devrions observer différentes relations entre différentes variables qui donnent davantage à voir une logique de prise de décision basée sur des réseaux informationnels intriqués plutôt qu'une logique de prise de décision basée sur des choix rationnels, systématiques liés par des causalités univoques et linéaires.

Si la perspicacité que nous prêtons aux chercheurs de la DATAR, dans ce que nous repérons comme un glissement sémantique porteur d'une autre conception de la transformation du tissu industriel, est valide, alors nous devrions repérer que les acteurs sociaux produisent des représentations qui témoignent notamment d'un rapport dialectique entre les informations qui structurent leurs représentations du changement. Dans le même esprit, nous devrions observer des représentations composites de leurs perspectives d'avenir, de leur destin en relation avec un contexte économique général qui s'internationalise; nous devrions caractériser un rapport relativement logique entre les liens qu'ils entretiennent avec les organismes d'État qui peuvent leur venir en aide et la manière dont ils se représentent le changement, le destin et l'avenir de leur entreprise; nous devrions observer des variations de représentations selon les secteurs de l'industrie, des récurrences dans les représentations indépendamment de ces secteurs à cause de l'interférence avec le milieu et nous devrions noter des variations entre les organismes selon les contraintes de l'environnement. Si notre hypothèse de la dualité homogénéisation et différenciation est juste, nous devrions, dans 
l'ensemble des représentations des responsables des entreprises, pouvoir comprendre comment, d'un côté, le monde industriel se globalise alors que, de l'autre côte, il se parcellise? La pensée systémique et complexe invite à se représenter non pas des oppositions entre ces catégories d'hypothèse et de pensée mais des conjonctions qui, sans cesser d'être quelquefois antinomiques, favorisent l'émergence d'inédits. Dans ce maelström des représentations, nous devrions observer que la formation elle-même est une ressource au service de la production de représentations pointillistes, nuancées. Nous faisons l'hypothèse en effet que la formation est une ressource mobilisable dans le cadre du projet d'adaptation industrielle des entreprises. La formation est une réponse possible du système "entreprise » dans le concert de la mondialisation et de la postmodernité. Elle est un opérateur de complexité.

Telles sont quelques-unes des hypothèses que nous tentons de vérifier. Nous le faisons en nous appuyant sur les résultats obtenus à partir d'une collecte de données faite auprès de porteurs de projets de la région MidiPyrénées. Nous entendons présenter ces résultats au travers d'une systémique des variables ou des phénomènes, ou encore des opinions. Nous entendons par systémique une manière d'aborder les données, une manière de sortir de la simple analyse unidirectionnelle des phénomènes. Il nous semble que, au-delà des corrélations strictes qu'on établit entre les variables, il est possible de modéliser les résultats de telle manière qu'on aperçoive comment les analyses qui pourraient être considérées comme parallèles, au fond, se combinent pour constituer un ensemble plus ou moins intégré d'informations. Nous souhaitons montrer que l'interprétation peut faire surgir logiquement ce qui échappe à la simple analyse empirique et qui, pourtant, se révèle aisément à l'intuition.

Il est intéressant de constater, par exemple, que les chefs d'entreprise, de manière intuitive, par l'importance qu'ils accordent aux divers éléments qui, selon eux, caractérisent le changement, rendent compte d'une manière de penser autour des notions de système et de lien. Il est tout aussi remarquable que les porteurs de projets d'adaptation soumettent la production aux champs communicationnel et commercial. Tout se passe comme si la représentation du changement et des actions d'adaptation qui lui sont liées est émergente d'une vision composite.

L'analyse des données recueillies montre que les chefs d'entreprises, loin d'avoir une vision univoque du changement et des actions d'adaptation à entreprendre, oscillent entre deux positionnements perméables l'un à l'autre. La plupart des variables étudiées montrent que la détermination des actions d'adaptation est réelle sans être, pour autant, complète, c'est-à-dire qu'elle est attribuable de manière non aléatoire à certains facteurs, mais que ces facteurs 


\section{NPSS, VOLUME 1, NUMÉRO 1, 2005}

n'expliquent pourtant pas à eux seuls cette détermination et qu'il est nécessaire d'y ajouter d'autres facteurs qui, sans être prépondérants, n'en sont pas moins importants dans la compréhension du phénomène. Les chefs d'entreprise oscillent ainsi entre des tendances contradictoires - homogénéité et différenciation, intégration et exclusion - quil faut se représenter comme autant de dialogiques qui structurent les représentations et les actions d'adaptation.

Notre contribution consiste à argumenter modestement en quoi l'entremêlement paradoxal, quelquefois antinomique, des hypothèses de la mondialisation et de la postmodemité ourdit les processus d'adaptation des entreprises et tisse leurs stratégies.

Nous proposons dans un premier temps de préciser qu'elle a été notre méthode de construction de l'échantillon de base de nos analyses. Nous présenterons ensuite, en lien avec les questions qui nous préoccupent, certains des résultats qui nous semblent les plus illustratifs.

\section{Méthode de construction de l'échantillon}

Notre contribution s'appuie sur les données recueillies dans le cadre d'un programme de recherche plus global qui consistait à évaluer sur le territoire français l'impact des financements européens (FSE-ObjectifIV) conjugués aux financements nationaux (privés et publics) sur les projets des entreprises. Une recherche nationale était diligentée par le Ministère du Travail et coordonnait différentes équipes de chercheurs sur le terrain de plusieurs régions françaises, dont la Région Midi-Pyrénées?

Durant la période de 1996 à 2001, le Fonds Social Européen et le Ministère du travail, de l'emploi et de la formation professionnelle français ont conjointe-

\footnotetext{
${ }^{7}$ La Région Midi-Pyrénées est la plus grande région française par sa taille. Elle se situe dans le sud de la France. Frontalière avec l'Espagne, elle compte huit départements essentiellement ruraux et un grand pôle d'activité, Toulouse, capitale régionale. Au dernier recensement, elle compte 2613000 habitants (1 274000 hommes et 1339000 femmes) dont $4 \%$ sont âgés de moins de vingt ans, $52 \%$ ont entre vingt et cinquanteneuf ans et $24 \%$ ont plus de soixante ans. Tous secteurs confondus, 131705 entreprises sont réparties sur le territoire de Midi-Pyrénées (4,6\% du nombre d'entreprises françaises), 45951 entreprises artisanales $(5,4 \%$ du nombre d'entreprises françaises). Cela représente un taux de création d'activité de 10,8\% alors que le taux national est de 10,9\%. En Midi-Pyrénées, les petites entreprises industrielles et artisanales contribuent de façon significative à l'activité industrielle. Elles sont au nombre de 8200 dans la région Midi-Pyrénées, rassemblant plus de 30000 personnes, soit un emploi industriel sur cinq. En effectif, la région Midi-Pyrénées se place ainsi au cinquième rang des régions françaises après Ile-de-France, Rhône-Alpes, ProvenceAlpes-Côte d'Azur et Aquitaine.
} 
ment accompagné, sur le seul territoire de Midi- Pyrénées, 456 projets d'entreprise dans une dynamique d'adaptation industrielle.

De manière assez classique, une première approche de notre problématique a consisté, au travers d'une exploration documentaire, en l'appropriation des dispositions réglementaires et des différents travaux menés sur cette question. Cette approche, complétée par une première investigation du terrain (trente dossiers étudiés, dix-sept interviews d'acteurs institutionnels et de porteurs de projets) a permis de nourrir notre problématique et de développer diverses orientations pour l'analyse. Afin d'analyser l'impact des financements européens (FSE-Objectif IV) conjugués aux financements nationaux sur les projets des entreprises de Midi-Pyrénées et, donc, de structurer le recueil d'informations, nous avons retenu dix unités d'information qui définissent une typologie qualitative, c'est-à-dire une typologie basée sur le repérage de formes situationnelles caractérisant un scénario. Il s'agit en l'occurrence de l'ensemble des facteurs élémentaires, caractéristiques et déterminants des projets cofinancés par le FSE-Objectif IV: identification du porteur de projet ${ }^{8}$, le porteur de projet et les changements de son environnement ${ }^{9}$, le porteur de projet et sa stratégie ${ }^{10}$, le

\footnotetext{
${ }^{8}$ Dans cette unité d'information, il s'agit de repérer l'identité du porteur de projet. Nous repérons le secteur d'activité du porteur de projet, sa raison sociale, la fonction de la personne en charge du projet, la date de création de la structure, l'effectif de la structure, la répartition par catégorie socioprofessionnelle, la structuration de la représentation du personnel, la proximité de l'entreprise par rapport à son marché.

${ }^{9}$ Les informations recherchées dans cette unité d'information sont relatives aux représentations et aux pratiques actuelles du porteur de projet vis-à-vis des changements de son environnement. Nous repérons les opinions concernant les perspectives de changements, l'inscription temporelle de ces changements, les niveaux et la caractérisation des changements, le niveau d'information par rapport à ces changements, les moyens d'information utilisés pour se faire une opinion sur les changements.

${ }^{10}$ Les informations recherchées dans cette unité d'information sont relatives aux représentations et aux pratiques actuelles du porteur de projet vis-à-vis d'un fonctionnement dans un mode stratégique. Nous repérons l'intérêt déclaré par le porteur de projet concernant les avantages de se doter d'une stratégie, les personnes concernées par la construction de la stratégie, l'implication plus ou moins importante de toutes les catégories de personnel, les éléments clés de la stratégie annoncée, le niveau de structuration de cette stratégie, l'articulation de cette stratégie à des plans d'action.
} 
porteur de projet et sa fonction «Ressources Humaines " " , le porteur de projet et sa fonction «Formation " ${ }^{12}$, les informations sur l'Objectif IV du FSE ${ }^{13}$, l’appui technique pour élaborer le projet ${ }^{14}$, les démarches administratives ${ }^{15}$, le

${ }^{11}$ Les informations recherchées à ce niveau sont relatives aux représentations et aux pratiques actuelles de l'entreprise vis-à-vis de cette fonction ainsi qu'aux évolutions qu'a éventuellement connues cette fonction en rapport avec le projet cofinancé par l'objectif IV. Nous repérons l'existence de cette fonction, la personne en charge de cette fonction, les activités liées à cette fonction, la relation avec le projet cofinancé, la modification éventuelle de cette fonction en relation avec le projet cofinancé.

12 Les informations recherchées dans cette unité d'information concernent les représentations et les pratiques actuelles de l'entreprise vis-à-vis de la fonction «Formation» ainsi que les évolutions qu'a éventuellement connues cette fonction en rapport avec le projet cofinancé par l'Objectif IV. Il s'agit également de repérer selon quelle optique l'entreprise établit son ou ses plans de formation: niveau stratégique ou niveau opérationnel. Nous repérons l'existence de cette fonction, la personne en charge de cette fonction, les activités liées à cette fonction, la relation avec le projet cofinancé, la modification éventuelle de cette fonction en relation avec le projet cofinancé.

${ }^{13}$ Il s'agit ici d'explorer par quels moyens (humains et techniques) le porteur de projet a eu connaissance des possibilités de l'Objectif IV ainsi que de qualifier (accessibilité, qualité) l'information reçue. Nous repérons la structure qui a informé le porteur de projet, la nécessité de recourir à des informations complémentaires, la qualité de l'information reçue, le niveau d'information sur l'origine du cofinancement; les difficultés rencontrées lors de la recherche d'information.

${ }^{14}$ Cette unité d'information permet d'explorer la qualité des appuis techniques reçus, de recueillir l'avis des bénéficiaires sur ces appuis. Nous repérons le recours à un appui technique de la part du porteur de projet, le souhait d'être aidé dans le montage du dossier, la structure d'appui, le niveau d'appui technique, la facturation éventuelle de cet appui, l'apport le plus important de l'appui technique, les collaborations ultérieures éventuelles avec les structures d'appui.

${ }^{15}$ Il s'agit ici de recueillir l'avis des porteurs de projet sur les aspects administratifs. Nous repérons l'opinion du porteur de projet sur l'accessibilité, la compréhension du dossier de demande de concours, les effets induits par la constitution du dossier, les relations avec la cellule FSE de la DRTEFP, les circuits administratifs de dépôt du dossier, le niveau de satisfaction par rapport aux délais de traitement administratif des dossiers et des paiements, les difficultés rencontrées sur les aspects administratifs. 
plan de formation cofinancé ${ }^{16}$, les effets du cofinancement FSE et du plan de formation ${ }^{17}$.

Ces dix unités d'information se retrouvent naturellement dans le questionnaire (outil central utilisé dans notre recueil d'informations) et sont déclinées à travers 154 questions dont certaines ont plus de vingt modalités de réponses possibles. Ces modalités de réponses ont été, pour la grande majorité d'entre elles, aperçues au fil de dix-sept interviews menées dans l'étape de construction de la problématique. Ce questionnaire a été envoyé aux 456 porteurs de projets qui sont les répondants aux questionnaires (chef d'entreprise ou cadre chargé de la conception et de la mise en ouvre du projet). Au terme de notre recherche, 183 questionnaires ont été retenus et ce sont eux qui constituent notre échantillon.

\section{La contribution du FSE}

Plusieurs questions se posent en vertu des données dont nous disposons sur la représentation qu'ont les répondants de la contribution du FSE à leur projet. Nous disposons également de données sur la manière dont les répondants

\footnotetext{
${ }^{16}$ Les différents éléments suivent une logique d'ingénierie de la formation qui, de la phase amont (préparation et conception du projet) à la phase aval (résultats et évaluation), en passant par la phase de réalisation, permet de repérer l'année de demande de concours, les scénarios de cofinancement, les événements à l'origine du projet, le contexte financier du porteur de projet au moment du cofinancement, les effets induits par la réalisation du dossier de demande de concours, les difficultés dans l'élaboration, le niveau de participation des salariés, les modalités de consultation des salariés, les difficultés rencontrées lors de la consultation des salariés, les dates de début et de fin du projet, les décalages éventuels et leurs causes, les bénéficiaires ultimes (salariés) des actions, les critères qui ont prévalu dans le choix du prestataire extéricur au porteur de projet, le niveau de formalisation de la proposition du prestataire, le niveau de satisfaction par rapport au déroulement du projet et ses retombées, les acquis applicables et transférables, les implications de ce projet sur la structuration des fonctions « Ressources Humaines » et "Formation ».

${ }^{17}$ Dans cette unité d'information, il s'agit d'explorer les effets du cofinancement FSE et, plus largement, les effets induits du projet au-delà des acquis immédiats. Nous repérons les opinions sur la contribution du FSE, le niveau d'enrichissement au projet initial amené par le cofinancement, le degré de satisfaction par rapport aux actions développées dans le projet et la qualité de la réalisation du projet, le caractère innovant et l'amplitude du projet, la contribution du projet à la structuration des fonctions «Ressources Humaines » et «Formation», les effets du cofinancement sur la réflexion stratégique et sur le climat social, la contribution à l'obtention d'une certification, les effets déclarés sur la compétitivité de la structure et sur ses capacités d'anticipation, les effets du cofinancement sur l'exclusion des salariés.
} 
perçoivent le changement dans leur entreprise. Or, nous pouvons nous demander si la façon de concevoir le changement des répondants, de concevoir ce à quoi est soumis l'entreprise, ce vers quoi elle tend, a une influence sur la perception de la contribution du FSE à leur entreprise, autrement dit si la perception de la contribution du FSE dépend de la conception du changement.

Pour mesurer la conception du changement, le questionnaire comporte vingt-deux indices. Ces indices ont la forme d'énoncés et les répondants se prononcent sur chacun d'eux selon une échelle de Likert à quatre niveaux qui vont de « de manière incontoumable » (1) à « de manière insignifiante » (4).

\subsection{Analyse de facteurs et analyse de regroupement}

Dans une première intuition, nous nous sommes demandés si des positions relatives à l'ensemble de ces indices pouvaient se dégager des formes communes. Par exemple, il nous a semblé que certaines réponses pouvaient être regroupées autour de thématiques comme la préoccupation pour les ressources humaines, ou pour la concurrence internationale, ou pour la fonction commerciale... Pour le vérifier, nous avons procédé à une analyse de facteurs. Cette analyse a dégagé six facteurs dont la valeur Eigen est d'au moins un. Ces six facteurs, toutefois, ne contiennent que $60,8 \%$ de l'ensemble de la variance qui appartient aux variables originales. Il est donc abusif de substituer ces six facteurs aux vingt-deux variables originales. Cependant, les facteurs qui ont été extraits permettent de jeter une certaine lumière sur les objets qui préoccupent ou non les répondants.

Le premier facteur réunit les positions où le commerce se veut peu déterminant; les répondants craignent peu les changements de la clientèle, n'ont pas le sentiment de devoir optimiser la fonction commerciale ou de diversifier les produits. Le deuxième facteur est du même ordre quoiqu'il ait trait davantage à la macroéconomie; il se rapporte à une situation peu affectée par la mondialisation des échanges économiques, par la concurrence internationale ou par l'évolution technologique des machines. Le troisième, lui, se rapporte à la communication et au personnel: le changement, là, se caractérise peu, aux yeux des répondants, par la mise en place d'un système informatique ou d'un système de communication exteme, d'une part, et par la gestion prévisionnelle des emplois et des compétences ou par l'évaluation des compétences des salariés, d'autre part. Le quatrième facteur renvoie à un faible questionnement sur le rôle des changements de direction ou sur un éventuel plan social. Le cinquième manifeste une insistance sur l'aménagement et la réduction du temps de travail et, corrélativement, il témoigne du peu d'importance accordée au renforcement des relations avec les donneurs d'ordres. 


\section{Tableau 1}

Analyse de facteurs pour les variables relatives à la conception du changement Poids, après rotation orthogonale, de chacune des variables dans chacun des facteurs quand elle est $\geq 0,30$

Énoncés :

Poids pour chacun des facteurs

Pour votre entreprise, aujoutd'hui, le changement se caractérise par :

Un haut degré de mondialisation des échanges économiques

Des relations renforcées avec les donneurs d'ordres

Des relations avec les sous-rraitants

Des relations avec les entreprises ayant des activités complémentaires à la production de l'entreprise

La mise en place d'un système infomatique en réseau pour l'ensemble des fonctions de l'entreprise

La mise en place d'un nouveau système

La réorganisation du travail pout augmenter

I.évaluation nécessaire des compćtences des salariés

\begin{tabular}{|c|c|c|c|c|c|c|}
\hline Variance & 13 & 13 & 12 & 9 & 8 & 6 \\
\hline Variance cum ulative & 13 & 26 & 38 & 47 & 55 & 61 \\
\hline Valeur Eigen & 295 & 283 & 261 & 200 & 164 & 134 \\
\hline
\end{tabular}


Le sixième facteur, enfin, correspond à une faible inquiétude pour ce qui est du vieillissement de la population salariée. Il importe toutefois de retenir que ces facteurs laissent échapper $39 \%$ de la variance qui est contenue dans les variables initiales et que, par conséquent, il est sage de faire porter l'analyse sur la totalité de ces variables, plutôt que sur les seuls facteurs. Quoi qu'il en soit, il semble qu'il y ait des types d'attitudes au sein de l'ensemble des répondants. L'analyse de facteurs fait apparaître une représentation du changement plus nuancée qu'il n'y paraît de prime abord. Dans une conception classique, nous pourrions nous attendre à une construction de la représentation globalement disjonctive du changement. Le changement est ceci ou cela. Or, il n'y a pas d'attitudes très tranchées. Un ensemble de dimensions participe de la représentation du changement. Si le changement est à la fois peu caractérisé par « les changements de la clientèle ", " la fonction commerciale », «la diversification les produits», «la mondialisation des échanges », " les changements de direction »... cela signifie qu'il est donc aussi caractérisé par ces dimensions. Le changement est un maillage de dimensions qui sont, de manière complémentaire et contradictoire, interactives. L'influence des dimensions prises une à une n'est pas significative, mais l'ensemble interagissant donne peut-être du sens aux conduites des entreprises. Cette représentation du changement a aussi ceci de paradoxal que tout change et rien ne change. Tout change un peu, mais pas suffisamment pour pouvoir attribuer le changement à une ou deux dimensions. Du coup, rien ne change beaucoup et pourtant ces entreprises ont choisi délibérément de s'engager dans un projet d'anticipation des mutations industrielles. La seule certitude est que nous assistons à la fin de la certitude d'une représentation du changement univoque. Le changement est incertitude tant par sa construction (un ensemble de dimensions en interactions plutôt que des dimensions exclusives les unes des autres) que par son activité (il est difficile d'attribuer une conduite d'entreprise à une telle représentation multidimensionnelle).

Toujours en rapport à la perception du changement, nous nous sommes demandés s'il était possible de réunir non pas les variables elles-mêmes, mais plutôt les répondants à partir des variables. Pour le découvrir, nous avons recouru à l'analyse de regroupement. L'analyse a construit deux grandes familles. Sauf pour les variables « la mise en place d'un système de qualité ", " le vieillissement de la population salariée " et "l'élévation nécessaire des compétences des salariés » où les centres des deux familles sont égaux, les centres de la première famille (59 individus) sont supérieurs à ceux de la seconde ( 87 individus) (37 cas sont exclus). Cela témoigne d'une plus grande préoccupation pour les indices dans la deuxième famille. Peu nuancée qu'elle est, cette analyse ne permet pas d'éviter de travailler 
avec les données originales, certes, mais elle renforce l'idée que nous avons justement une représentation mouvante et polycentrique à l'origine des actions engagées par les entreprises. Loin de pouvoir de manière exclusive attribuer telle action à telle représentation du changement, nous sommes obligés de construire une contribution du FSE comme constitutive d'une représentation polycentrique du changement.

\subsection{Perception du changement et contribution du FSE}

Pour examiner le lien entre la perception du changement et la contribution du FSE, nous avons procédé à des analyses de régression. Cette opération est statistiquement possible puisque les variables qui ont trait à l'apport du FSE, tout comme les perceptions des caractéristiques du changement, sont de nature cardinale. La méthode utilisée est celle de la sélection par tâtonnement (stepwise dans le logiciel SPSS) des énoncés se rapportant à la perception du changement dont l'influence est significative $(p<0,05)$ sur les variables qui concernent le FSE.

Vingt analyses ont ainsi été effectuées (chacune d'elles sur chacun des apports probables du FSE) impliquant chaque fois vingt-deux variables indépendantes, soit les opinions sur le changement. Dans dix-neuf de ces analyses, des énoncés relatifs aux caractéristiques du changement ont été sélectionnés. Les équations de régression qui apparaissent contiennent entre une et six variables: cinq fois une variable, quatre fois deux variables, six fois trois variables, une fois quatre variables, deux fois cinq variables et une fois six variables. Les variances expliquées, par la perception du changement, de la manière dont est conçu l'apport du FSE ont tendance à s'accroître avec le nombre de variables qui composent l'équation: elles vont de $3 \%$ à $38 \%$; pour les dix-neuf analyses, elles sont en moyenne de $14 \%$. Elles sont donc faibles dans l'ensemble, ce qui permet de conclure que la façon de comprendre la contribution du FSE est associée, mais légèrement, à la perception des caractéristiques du changement. Là encore, pas de causalité univoque, pas de caractérisation linéaire, pas de repérage prédictif; néanmoins des tonalités, des mouvances.

La plupart des corrélations sont positives. Cela signifie que moins la caractéristique du changement se révèle importante à ses yeux, moins le répondant tend à considérer que la contribution du FSE a été utile. Il y a toutefois des corrélations négatives. Nous remarquons que si le changement se caractérise peu par le système de communication, alors l'apport du FSE se distingue; c'est le cas pour la compétitivité de l'entreprise, l'accélération de l'obtention d'une certification qualité, le renforcement du partenariat avec les sous-traitants, le renforcement du partenariat avec les donneurs 
d'ordres, l'accroissement des gains de productivité et l'obtention de nouveaux marchés. Comme si le système de communication ne posait que peu de questions pour l'entreprise (le changement se caractérise peu par le système de communication) tout en posant quand même suffisamment de questions pour amener l'entreprise à agir de manière anticipative à d'éventuels problèmes qui seraient davantage conséquents! Ici, le système de communication est globalement envisagé comme fiable et pertinent, validé par le fonctionnement de l'entreprise. Le FSE vient alors pour renforcer la dynamique engagée et permettre d'aller plus loin dans cette logique. Il renforce l'existant.

On note aussi qu'un changement peu défini par les nouveaux produits favorise l'apport du FSE sur l'accroissement de productivité, la possibilité d'accéder à des formations qualifiantes, la valorisation des emplois existants et la clarification du rôle des différents services internes. Comme précédemment, le marché semble valider la stratégie " produits » de l'entreprise puisque le changement est peu caractérisé par les nouveaux produits. Cette base de fonctionnement permet alors à l'entreprise de dégager du temps pour accroître sa productivité, accéder à la formation, valoriser les emplois, clarifier les apports des uns et des autres dans la chaine de production. L'apport du FSE participe d'un cercle vertueux. Le marché valide la pertinence des produits, le FSE accompagne l'entreprise dans son fonctionnement. D'une part, il permet l'accroissement de la valorisation des composantes internes productrices de cette pertinence: la ressource humaine; et, d'autre part, il accompagne l'entreprise dans la création de nouveaux ancrages sur le marché: optimisation de la fonction commerciale, lancement de nouveaux produits.

Certaines caractéristiques du changement se distinguent: « la mise en place d'un nouveau système de communication externe » se manifeste six fois; «la mise en place d'un système informatique en réseau pour l'ensemble des fonctions de l'entreprise », cinq fois; «la mise en place d'un système qualité » et « des incertitudes liées au lancement de nouveaux produits », quatre fois.

Les chefs d'entreprise, de manière intuitive, par l'importance qu'ils accordent à divers éléments, rendent compte d'une manière de penser autour des notions de système et de lien. Il est fortement question de système de communication externe et de système d'informatique pour les fonctions de l'entreprise. Il y a là une dialogique dans l'esprit des administrateurs des entreprises où la dynamique interne et le rapport à l'environnement extérieur agissent l'un par rapport à l'autre pour déterminer la manière de concevoir le devenir de l'entreprise. 


\section{Tableau 2}

Régression multiple de toutes les variables relatives à la représentation actuelle du changement $(1=$ de manière incontournable et $4=$ de manière insignifiante) dont la contribution est apparue significative pour les variables relatives à la manière de concevoir l'apport du FSE ( 1 = tout à fait d'accord et $4=$ pas du tout d'accord $)$. Coefficient standardisé $(\beta)$ et variance expliquée $\left(\mathrm{R}^{2}\right)$

\begin{tabular}{|c|c|c|}
\hline $\begin{array}{l}\text { Variables relatives à la manière de concevoir } \\
\text { l'apport du FSE }\end{array}$ & $\begin{array}{l}\text { Variables relatives à la représentation } \\
\text { actuelle du changement }\end{array}$ & $\beta$ \\
\hline $\begin{array}{l}\text { Une capacité supplémcntairc d'anticipation } \ldots \ldots \ldots \ldots \ldots \\
\text { pour la conduite de l'entreprise }\end{array}$ & $\begin{array}{l}\text { Gestion des emplois et des compétences } \\
\text { Anénagement du temps de travail } \\
\text { Vieillissement de la population des } \\
\text { salariés }\end{array}$ & $\begin{array}{l}0,17 \\
0,18 \\
0,17\end{array}$ \\
\hline
\end{tabular}

environnement

De meilleures garanties pour la pérennisation ........ Fusion avec d'autres cntites ..........

de l'entreprise

Des atouts pour augmenter la compéritivité ........ Système informatique ......... 0,28

$\begin{array}{lr}\text { Systeme de communication } & 0,25 \\ \text { Syles salariés . } & -0,22\end{array}$

Une accélération de l'obtention d'une certification $\ldots \ldots . \quad$ Système qualité ............. 0.41

Système qualité ............... 0,41
Donneurs d'ordres

qualité

Plan socia!

0,21

Système de communication

$-0,20$

Un développement du réseau de partenaires

Système qualité .............

à l'entreprise

Un renforcement du partenariat avec . . . . . . . . .

le ou les sous-traitants

Sous-traitants $\ldots \ldots \ldots \ldots \ldots \ldots \ldots, 0 \ldots \ldots$

Systeme de communication

$\begin{array}{lr}\text { Systeme de communication } & -0,38 \\ \text { Système informatique } & 0,19\end{array}$

Changement de direction $\quad 0,18$

Aménagement du temps de travail $\quad 0,16$



$\begin{array}{ll}\text { le ou les donneurs d'ordres } & \begin{array}{l}\text { Donncurs dordris } \ldots \ldots \ldots \\ \text { Système de communication }\end{array}\end{array}$

$\begin{array}{lr}\text { Système de communication } & -0,37 \\ \text { Aménagement du temps de traval } & 0,20\end{array}$

Fusion avec d'autres critités $\quad 0,21$

Système informatique 0,18

Un accroissement des gains de productivité

Réorganisation du travail ......... $\quad 0,41$

Fonction commerciale $\quad 0,26$

Systcme de communication $\quad-0,21$

Donneurs d'ordres $\quad 0,13$

Nouvcaux produits $\quad-0,20$

$\begin{array}{lr}\text { Mondialisation } & 0,15\end{array}$

Le maintien sur les marchés existants $\ldots \ldots \ldots \ldots \ldots \ldots$ Clientèle $\ldots \ldots \ldots \ldots$

Système informatique $\quad 0,25$

L'obtention de nouveaux marchés $\ldots \ldots \ldots \ldots \ldots \ldots$ Fonction commercialt $\ldots \ldots \ldots$

Système informatique $\quad 0,29$

Système de communication $\quad-0,26$

lílévation des compétences des salariés . . 0,25

Une aide au dialogue social interne à l'entreptisc . . . . . . .

Réotganisation du travail . . . . . . . 0,18

Système qualité . . . . . $\ldots \ldots \ldots$

Sous-traitants
Elevation des com

Élévation des compétences des salariés. $\quad 0,30$

Plan social $\quad-0,19$

Mondialisation ............. 0,24

Élévation des compétences des salarićs $\quad 0,18$

Nouveaux produits ........... $\quad-0,32$

Changement de direction $\quad 0,22$

Système qualité $\quad 0,10$

Gestion des cmplois et des compétences. $\quad 0,30$

Noureaux produits $\quad 0,24$

$\begin{array}{lr}\text { Fusion avec d'auttes entités } & 0,18\end{array}$

Plan social .............. 0,23

La clarification du róle des différents

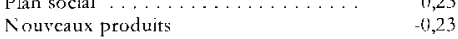

Fonction commerciale $\quad 0,21$

Le maintien de P'emploi.

Changcment de direction 
Il faut signaler l'absence de caractéristiques du changement: «l'intensité de la concurrence internationale », par exemple. Il est toutefois permis de croire que l'incidence du «haut degré de mondialisation des échanges économiques » est telle qu'elle écarte la première caractéristique puisque ces deux aspects sont fortement corrélés. D'autres éléments, qui ont trait, cette fois, à la production, ne trouvent aucun espace dans les équations de régression: diversification des produits, évolution des machines, transformation des techniques de fabrication, relation aux entreprises complémentaires à la production. Cela renforce lidée que l'entreprise n'est pas en crise au sens habituel du terme (perte de repère, perte financière...) mais au sens premier du terme de mobilisation en vue d'un futur incertain. Cela signifie sans doute que les questionnements liés à l'environnement, interne et externe, et à la commercialisation supplantent les réflexions qui se rapportent à la production. En fait, comme c'est souvent le cas dans les sociétés informationnelles, tout se passe comme si la production était soumise au champ communicationnel et au champ commercial. L'importance du champ commercial est signifiée par deux variables (optimisation de la fonction commerciale et incertitudes liées au lancement de nouveaux produits) que l'on retrouve sollicitées sept fois.

\section{Démarche qualité et contribution du FSE}

Le questionnaire comporte une série de six questions qui se rapportent au plan de formation et à la démarche qualité. Les responsables des entreprises y répondent par oui ou par non. Nous pouvons donc recourir à un test $t$ et vérifier s'il y a une différence selon la réponse qui est donnée pour les diverses contributions du FSE.

La première question est la suivante: « le plan de formation s'inscrivait-il dans une démarche qualité? ». Nous notons peu de variation selon la réponse qui est foumie. Pour quatorze des vingt aspects de l'apport du FSE, il n'y a aucune différence significative. Dans les autres cas, les différences sont faibles, ne dépassant pas la valeur de 0,58 sauf pour l'« accélération de l'obtention d'une certification qualité » ou l'écart est de $1,24^{18}$. Chaque fois, la moyenne est supérieure pour l'ensemble des personnes qui répondent « non », c'est-à-dire que si le plan de formation ne s'inscrit pas dans une démarche qualité, les responsables des entreprises sont moins enclins à parler d'un apport du FSE. Cela invite à penser que le FSE vient renforcer une dynamique de l'entreprise et accompagne un cercle vertueux de l'anticipation de la mutation.

${ }^{18} \mathrm{t}_{(141,95)}=8,93 ; \mathrm{p}<0,05$. 
La deuxième question est formulée ainsi: «Depuis ce plan de formation, êtes-vous certifié? » Cette fois, nous ne découvrons de différence que pour une seule dimension de l'apport du FSE: l'« accélération de l'obtention d'une certification qualité » et la moyenne est supérieure une nouvelle fois pour les personnes qui ont répondu de façon négative $(2,87 \text { pour } 2,00)^{19}$. Cela est on ne peut plus logique: si nous ne sommes pas certifiés, alors nous tendons moins à dire que le FSE a accéléré l'obtention d'une certification qualité ».

La troisième question se lit comme suit « Si oui, ce plan de formation a-t-il contribué à l'obtention de la certification? » 11 y a alors deux lieux de différence significative, l'un pour « aide à la réalisation du plan de formation $~^{20}$ et, l'autre, encore pour " accélération de l'obtention d'une certification qualité $»^{21}$; les moyennes sont toujours supérieures pour les personnes qui répondent «non ».

La quatrième question demande: "Le plan de formation cofinancé s'inscrivait-il dans une action de réorganisation du travail? » Quatre aspects de la contribution du FSE présentent des différences inférables: « des atouts pour augmenter la compétitivité de l'entreprise $»^{22}$, «un renforcement du partenariat avec le ou les sous-traitants $»^{23}$, « un accroissement des gains de productivité $»^{24}$ et « une amélioration du climat social $»^{25}$. Les différences de moyennes sont faibles et elles sont toujours à l'avantage des positions négatives.

La cinquième question prend cette forme: «L'objectif a-t-il été atteint? » Trois aspects donnent lieu à des variations significatives " une meilleure adaptation de l'entreprise à son environnement " ${ }^{26}$, " de meilleures garanties pour la pérennisation de l'entreprise $»^{27}$ et " des atouts pour augmenter la compétitivité de l'entreprise $»^{28}$. Les moyennes sont quelque peu plus élevées chez les responsables d'entreprise qui répondent « non».

La sixième et dernière question permet de découvrir si « plus précisément, le plan de formation cofinancé s'inscrivait dans une démarche d'aménagement et de réduction du temps de travail (Loi Robien ou loi Aubry relative aux 35 heures hebdomadaires)? » Cinq des vingt aspects présentent des différences significatives: « une capacité supplémentaire d'anticipation pour la conduite

\footnotetext{
${ }^{19} \mathrm{t}_{(166)}=-4,48 ; \mathrm{p}<0,05$.

${ }^{20} \mathrm{t}_{(40)}=-2,13 ; \mathrm{p}<0,05$.

${ }^{21} \mathbf{t}_{(39)}=-3,68 ; \mathrm{p}<0,05$.

${ }^{22} \mathrm{t}_{(168)}=-2,35 ; \mathrm{p}<0,05$.

${ }^{23} \mathbf{t}_{(162)}=-2,45 ; \mathrm{p}<0,05$.

${ }^{24} \mathrm{t}_{(123,80)}=-3,17 ; \mathrm{p}<0,05$.

${ }^{25} \mathbf{t}_{(166)}=-2,29 ; \mathrm{p}<0,05$.

${ }^{26} \mathrm{t}_{(49,38)}=-2,40 ; \mathrm{p}<0,05$.

${ }^{27} \mathrm{t}_{(121)}=-2,01 ; \mathrm{p}<0,05$.

${ }^{28} \mathbf{t}_{(122)}=-2,34 ; \mathrm{p}<0,05$.
} 
de l'entreprise $»^{29}$, « une meilleure adaptation de l'entreprise à son environnement $»^{30}$, « un renforcement du partenariat avec le ou les sous-traitants $»^{31}$, "un accroissement des gains de productivité » ${ }^{32}$ et «l'obtention de nouveaux marchés $»^{33}$. Les moyennes sont plus élevées quand on répond «non ». La différence entre les deux groupes est plus faible pour l'adaptation à l'environnement $(0,33)$; elle est plus élevée pour l'obtention de nouveaux marchés $(0,64)$; ailleurs, elle se situe entre ces deux valeurs.

Nous pouvons donc conclure que, d'une façon générale, la population est plutôt homogène; il y a beaucoup plus d'égalités entre les moyennes des deux groupes que de différences. Quand il y a des différences, elles sont, la plupart du temps, faibles.

\section{Critère qui a prévalu dans le choix de l'organisme de formation et contribution du FSE}

Nous pouvons soulever l'hypothèse que le critère d'après lequel une entreprise a choisi un ou des organismes de formation influe sur les représentations qui sont relatives à la contribution du FSE. Le questionnaire contient huit informations qui permettent d'observer ce critère. Ces informations peuvent être associées aux divers indices de l'apport du FSE.

Les répondants sont d'abord appelés à choisir parmi un ensemble de critères qui leur sont proposés: la "notoriété ", l'« antériorité d'une prestation », les " recommandations par des entreprises partenaires », les «recommandations de l'OPCA », les « recommandations de la fédération professionnelle », le " choix du donneur d'ordres », " le choix du groupe auquel vous appartenez ». Compte tenu du nombre important de catégories et de la taille relativement faible de l'échantillon, il est préférable, ici, de recourir à un test non paramétrique. Parmi les vingt tests effectués, six donnent lieu à des variations qui ne peuvent être attribuables au hasard. Ces variations se rapportent à l'accroissement des gains de productivité ${ }^{34}$, au maintien sur les marchés existants ${ }^{35}$, à l'obtention de nouveaux marchés ${ }^{36}$, à l'aide à la réalisation du plan de formation ${ }^{37}$, à la clarification du rôle des

\footnotetext{
${ }^{29} \mathrm{t}_{(171)}=-2,80 ; \mathrm{p}<0,05$.

${ }^{30} \mathrm{t}_{(171)}=-2,23 ; \mathrm{p}<0,05$.

${ }^{31} \mathrm{t}_{(160)}=-3,30 ; \mathrm{p}<0,05$.

${ }^{32} \mathbf{t}_{(45,96)}=-4,26 ; \mathrm{p}<0,05$.

${ }^{33} \mathrm{t}_{(168)}=-2,98 ; \mathrm{p}<0,05$.

${ }^{34}$ Test Kruskal-Wallis: $\chi_{(6)}^{2}=14,80 ; \mathrm{p}<0,05$

${ }^{35}$ Test Kruskal-Wallis: $\chi^{2}{ }_{(6)}=17,52 ; \mathrm{p}<0,05$.

${ }^{36}$ Test Kruskal-Wallis: $\chi_{(6)}^{2}=20,23 ; \mathrm{p}<0,05$.

${ }^{37}$ Test Kruskal-Wallis: $\chi_{(6)}^{2}=13,47$; $\mathrm{p}<0,05$.
} 
différents services internes ${ }^{38}$ et au maintien de l'emploi ${ }^{39}$. Dans les six cas, le score est supérieur quand le responsable de l'entreprise mentionne le " choix du donneur d'ordres », ce qui signifie que, sur ces aspects, l'apport $\mathrm{du}$ FSE semble moins déterminant quand le principal critère qui a prévalu au choix de l'organisme a été le choix du donneur d'ordres.

Le fait d'avoir ou non rédigé un cahier des charges influe-t-il sur la manière dont on perçoit l'influence du FSE? Le fait d'avoir ou non rédigé un cahier des charges agit-il sur la manière dont on se représente la contribution du FSE à la formation? D’une façon générale, la réponse est négative. Cela signifie que la perception de l'apport du FSE est indépendante du fait de rédiger ou non un cahier des charges. Ce résultat est intéressant, car il heurte l'idée souvent développée à la fois par les financeurs, les relais techniques et les porteurs de projets selon laquelle la rédaction d'un cahier des charges permettrait de mieux évaluer l'apport de l'action une fois qu'elle a eu lieu - cette idée que la mobilisation des acteurs en vue de la rédaction du cahier des charges favoriserait, voire amplifierait la prise de conscience de leurs besoins, de leurs attentes, des tenants et aboutissants du projet de formation; cette idée que la formalisation par l'écrit faciliterait par voie de conséquences l'appréciation accrue des effets du financement supplémentaire et de l'action. Or, il semble que, globalement, cela ne soit pas le cas. Nous notons toutefois deux exceptions: « une meilleure adaptation des salariés aux évolutions internes » et "un développement de l'employabilité des salariés $»^{41}$. Les personnes qui répondent « non » témoignent alors d'un moins grand accord quant à l'apport du FSE pour ces deux aspects.

On peut distinguer les répondants selon que la proposition de formation était «conçue et réalisée pour vous » ou qu’elle était « repérable dans l'offre existante de l'organisme ». Sur les vingt aspects de l'apport du FSE, nous ne découvrons de différence significative que dans trois cas selon ces deux possibilités de réponse: « une meilleure adaptation des salariés aux évolutions internes $»^{42}$, « un développement de l'employabilité des salariés $»^{43}$ et " une possibilité accrue d'accéder à des formations qualifiantes »" Dans les deux premiers, nous notons une impression plus forte de l'apport du FSE quand la formation a été conçue pour soi, dans le troisième, c'est le

\footnotetext{
${ }^{38}$ Test Kruskal-Wallis: $\chi_{(6)}^{2}=16,69 ; \mathrm{p}<0,05$.

${ }^{39}$ Test Kruskal-Wallis: $\chi_{(6)}^{2}=15,28 ; \mathrm{p}<0,05$

${ }^{40} \mathrm{t}_{(161)}=-2,16 ; \mathrm{p}<0,05$.

${ }^{41} \mathrm{t}_{(142,02)}=-2,76 ; \mathrm{p}<0,05$.

${ }^{42} \mathrm{t}_{(135,87)}=-2,72 ; \mathrm{p}<0,05$.

${ }^{43} \mathrm{t}_{(135,18)}=-2,40 ; \mathrm{P}<0,05$.

${ }^{44}{ }_{t_{(152)}}=3,59 ; p<0,05$.
} 
contraire. Il est intéressant de constater que, à l'inverse de la rédaction d'un cahier des charges, la construction d'une formation sur mesure est liée positivement à la perception de l'apport du FSE. Tout se passe comme si, pour les répondants, « une meilleure adaptation des salariés aux évolutions internes » et « un développement de l'employabilité des salariés » étaient liés au montage ad hoc d'une formation en lien avec leurs besoins. Ce résultat est intéressant en termes de préconisations en direction des organismes chargés d'accompagner les entreprises dans la traversée des mutations industrielles. En effet, il est plus profitable pour les entreprises qu'on les accompagne dans la construction d'une formation sur mesure que dans la rédaction d'un cahier des charges. En revanche, la formation sur mesure, par définition, ne correspond pas aux standards de la formation qualifiante reconnue par un diplôme. Ainsi, il va de soi que les répondants, quand ils estiment que la formation était « conçue et réalisée » pour eux, ne permettait pas «d'accéder à des formations qualifiantes ». Il y a là une incompatibilité structurelle que les processus de Validation des Acquis de l'Expérience devraient pourvoir dépasser (une formation sur mesure devrait pouvoir être prise en compte dans un processus de validation diplômant).

Que l'organisme de formation soit ou non intervenu en amont dans le montage du dossier, cela n'a pas réellement d'effet sur la façon d'apprécier le FSE. Les moyennes ne sont inégales que pour deux des vingt aspects et les différences sont faibles. Si l'organisme s'est impliqué préalablement, la contribution se veut légèrement moins bien perçue pour « une meilleure adaptation de l'entreprise à son environnement ${ }^{45}$; elle est un peu mieux perçue pour « une aide au dialogue social interne à l'entreprise » ${ }^{46}$.

Dans le questionnaire, on lit les questions suivantes: « dans tous les cas, pensez-vous que vos objectifs et vos attentes ont été compris? ", "vos objectifs et vos attentes ont-ils été pris en compte » et «les actions de formation qui vous ont été proposées étaient-elles adaptées? ». Trois réponses sont possibles: « complètement », " partiellement » et « pas du tout ». Personne n'a choisi la dernière réponse, pour aucune des trois questions. Nous pouvons donc effectuer des tests sur les différences de moyennes entre deux groupes. Encore une fois, les égalités dominent: seize sur vingt, quinze sur vingt et dix-sept sur vingt. En rapport à la première, il y a donc quatre lieux d'inégalités. Quand on répond « complètement», on est plus d'accord sur la contribution du FSE sur les trois aspects suivants: "des atouts pour augmenter la compétitivité de l'entreprise»", " une

\footnotetext{
${ }^{45} \mathrm{t}_{(167)}=2,62 ; \mathrm{p}<0,05$.

${ }^{46} \mathrm{t}_{(166)}=-3,21 ; \mathrm{p}<0,05$.

$\mathrm{t}_{(174)}=-2,37 ; \mathrm{p}<0,05$.
} 
meilleure adaptation des salariés aux évolutions internes $»^{48}$, «un développement de l'employabilité des salariés $"{ }^{49}$. L'accord est un peu plus élevé quand on répond partiellement pour « une aide au dialogue social interne à l'entreprise ${ }^{50}$. Relativement à la deuxième question, nous trouvons cinq différences inférables. Quatre d'entre elles témoignent d'une considération un peu plus forte pour l'apport du FSE quand on répond «complètement »: « une capacité supplémentaire d'anticipation pour la conduite de l'entreprise $~^{51}$, « une aide à la réalisation du plan de formation $~^{52}$, « une meilleure adaptation des salariés aux évolutions internes $"$ " , "un développement de l'employabilité des salariés $"^{54}$. La cinquième montre un plus grand accord pour l'énoncé quand la réponse est «partiellement» et il s'agit de l'« aide au dialogue social interne à l'entreprise $~^{55}$. La troisième question ne signale que trois inégalités de moyennes qui ne soient pas attribuables au hasard. Quand la réponse est " complètement ", l'assentiment est quelque peu plus prononcé pour deux énoncés: "une meilleure adaptation des salariés aux évolutions internes $»^{56}$ et « un développement de l'employabilitédes salariés »" il l'est un peu moins pour la proposition « une aide au dialogue social interne à l'entreprise $1{ }^{58}$. L'ensemble de ces résultats montre toute la pertinence de l'écoute des besoins, des attentes et des objectifs de l'entreprise. Il souligne toute l'importance de développer des processus d'audit au premier sens du terme. Des processus où l'intervenant se définit en lien avec ses compétences de tiers maïeuticien plutôt que d'expert de procédures (cahier des charges, normes qualité). Ces résultats révèlent l'efficience vécue par les répondants à la condition que, en amont, les actions construites procèdent davantage d'une écoute active, d'un montage ad hoc et sur mesure de propositions de formation. Quand les objectifs et les attentes ont été compris et pris en compte, les entreprises expriment leur satisfaction et attribuent à la contribution du FSE une part non accessoire dans cette satisfaction.

\footnotetext{
${ }^{48} \mathrm{t}_{(171)}=-2,56 ; \mathrm{p}<0,05$.

${ }^{49} \mathrm{t}_{(169)}=-2,89 ; \mathrm{p}<0,05$.

${ }^{50} \mathrm{t}_{(171)}=2,36 ; \mathrm{p}<0,05$.

${ }^{51} \mathrm{t}_{(170)}=-2,50 ; \mathrm{p}<0,05$.

${ }^{52} \mathrm{t}_{(170)}=-2,33 ; \mathrm{p}<0,05$.

${ }^{53} \mathrm{t}_{(59,90)}=-3,56 ; \mathrm{p}<0,05$.

${ }^{54} \mathrm{t}_{(168)}=-2,97 ; \mathrm{p}<0,05$.

${ }^{55} \mathrm{t}_{(74,(6))}=2,06 ; \mathrm{p}<0,05$.

${ }^{56} \mathrm{t}_{(170)}=-4,37 ; \mathrm{p}<0,05$.

${ }^{57} \mathrm{t}_{(168)}=-2,84 ; \mathrm{p}<0,05$.

${ }^{58} \mathrm{t}_{(70,78)}=2,49 ; \mathrm{p}<0,05$.
} 
Une dernière question a pour but de découvrir si les responsables des entreprises procèderaient de la même manière « si c'était à refaire ». $\grave{A}$ nouveau, les énoncés qui donnent à observer des variations sont rares: deux sur vingt. La position est plus favorable si la réponse est affirmative pour « une capacité supplémentaire d'anticipation pour la conduite de l'entreprise $»^{59}$ et pour « un développement de l'employabilité des salariés ${ }^{60}$.

Dans l'ensemble, nous trouvons beaucoup plus de lieux de non-différence. Les inégalités sont plutôt rares et ne révèlent pas d'écarts importants. Certains aspects de l'apport du FSE se manifestent assez souvent.

\section{Constat d'un développement après les formations et contribution du FSE}

Les responsables des entreprises sont amenés à indiquer si, "suite aux formations suivies par les salariés », ils ont « constaté un développement » de leurs compétences repérables au travers de neuf aspects. Il est permis de penser que leur opinion sur ces développements a quelque influence sur la façon de concevoir la contribution du FSE. Nous pouvons procéder à cette vérification en effectuant des analyses de régression multiple auxquelles nous demandons de sélectionner les variables dont l'influence est significative sur chacune des composantes du FSE.

Ces analyses révèlent alors que la variance expliquée de la contribution du FSE par les constats de développement oscille entre $0 \%$ et $26 \%$. Les corrélations sont toutes positives, sauf deux. Elles montrent donc, compte tenu de la façon dont les données ont été recueillies, que moins l'aspect du développement apparaît important, moins nous tendons à reconnaitre les contributions du FSE, ce qui tombe sous le sens. Mais il ne faut pas perdre de vue que les variances expliquées sont plutôt faibles.

Une variable s'impose: « des capacités pour intégrer des exigences de qualité »; nous la trouvons dans huit équations. Si le répondant note ici un développement, il tend à être d'accord sur l'apport du FSE en ce qui a trait à l'adaptation de l'entreprise à l'environnement, l'augmentation de la compétitivité, l'obtention de la certification qualité, le renforcement du partenariat avec les sous-traitants ou avec les donneurs d'ordre, l'accroissement des gains de productivité, le maintien de marchés existants et l'obtention de nouveaux marchés. Deux autres variables se manifestent à cinq reprises: " du profil d'emploi des salariés » et " des capacités de communication. Les corrélations sont toujours positives. Elles vont donc

\footnotetext{
5) $\mathrm{t}_{(16,))}=-3,00 ; \mathrm{p}<0,05$.

${ }^{60} \mathrm{t}_{(167)}=-3,04 ; \mathrm{p}<0,05$.
} 


\section{dans le même sens. Les regroupements de variables qu'on observe dans les équations, toutefois, sont aléatoires: ici les exigences de qualité vont avec l'autonomie, là, avec la communication ou avec la production.}

\section{Tableau 3}

Régression multiple de toutes les variables relatives à la représentation du développement suite aux formations des salariés $(1=$ très important et $4=$ pas important) dont la contribution est apparue significative pour les variables relatives à la manière de concevoir l'apport du FSE ( $1=$ tout à fait d'accord et $4=$ pas du tout d'accord $)$.

Coefficient standardisé $(\beta)$ et variance expliquée $\left(R^{2}\right)$ Variables relatives à la manière de concevoir
l'apport du FSE
Variables relatives à la représentation du développement suite aux formations des salariés

Une capacité suppiémentaire d'anticipation ...... Des capacités d'analyse des siruations de travail .... 0 pour la conduite de l'entreprise

Une meilleure adaptation de l'entreprise . . . . . Des capacités pour intégrer des exigences qualité ... 0,3

à son environnement De la polyvalence

De meilleures garanties pour la pérennisation $\ldots \ldots$ Des capacités d'autonomie $\ldots \ldots \ldots$

de l'entreprise

Des atouts pour augmenter la compétirivité ...... Des capacités pour intégrer des exigences qualité ... 0,32

de l'entreprise

Des capacités d'autonomie 0,24

Une accélération de l'obtention . . . . . . . . Des capacités pour intégrer des exigences qualité ... 0

d'une certification qualité

Un développement du réseau de partenaires . . . . Des capacités de communication . . . . . . . . . 0,28

à l'entreprise $\quad$ Des capacités à intégrer des objectifs de gestion 0,19

Un renforcement du partenatiat ave le ou ....... Des capacités pour intégrer des exigences qualité . . 0,22

les sous-traitants Des capacités à in tégrer des objectifs de gestion 0,20

Des capacités de communication $\quad 0,16$

Un renforcement du partenariat avec . . . . . . De la polyvalence . . . . . . . . . . . . 0,22

le ou les donneurs d'ordres $\quad$ Des capacités pour intégrer des exigences qualité $\quad . . . .20$

Un accroissement des gains de productivité $\ldots \ldots \ldots$ Des capacités pour intégrer des objectifs $\ldots \ldots \ldots, 43$

de production $\quad 0,28$

Des capacités pour integrer des exigences qualité $\quad-0,22$

Des capacités d'analyse des situations de travail

Le maintien sur les marchés existants ......... Des capacités pour intégrer des exigences qualité ...

Des capacités pour intégrer des exigences qualité ... 0,32

Des capacités à in tégrer des objectifs de gestion $\quad 0,22$

$\begin{array}{ll}\text { Une aide au dialogue social interne à l'entreprise } \ldots & \begin{array}{l}\text { Du profil d'emploi des salariés } \ldots \ldots \ldots \ldots \\ \text { Des capacités de communication }\end{array} \ldots, \ldots, 27\end{array}$

Une amélioration du climat social $\ldots \ldots \ldots \ldots \ldots$ Du profil d'emploi des salariés . . . . . . . . . . 0,21

Des capacités de communication $\quad 0,17$

Une aide à la réalisation du plan de formation ....

Des capacités de communication

Une meilleure adaptation des salariés aux ...... Des capacités de communication . ......... 0,24

évolutions internes $\quad$ Des capacités pour intégrer des objectifs 0,16

Des capacites pour integrer des objectifs
de production

Un développement de "l'employabilité $n \ldots \ldots \ldots$ De la polyvalence $\ldots \ldots \ldots \ldots \ldots \ldots \ldots \ldots \ldots \ldots$
des salariés

Une possibilité accrue d'accéder à des $\ldots \ldots \ldots \ldots$ Du profil d'emploi des salariés $\ldots \ldots \ldots \ldots$

formations qualifiantes $\quad$ Des capacités pour intégrer des objectifs $\quad-0,20$

de production

Des capacités d'analyse des situations de travail $\quad 0,19$

La valorisation des emplois existants $\ldots \ldots \ldots \ldots$ Du profil d'emplei des salariés $\ldots \ldots \ldots \ldots$

0

La clarification du rôle des différents $\ldots \ldots \ldots \ldots$ Du profil d'emploi des salariés $\ldots \ldots \ldots \ldots$

0




\section{8

Les constats sur les divers aspects du développement ne dépendent du fait que la formation s'inscrivait ou non dans une démarche qualité que pour un indice: «des capacités pour intégrer des exigences qualité », et il va sans dire que si tel était le cas, le niveau d'importance est plus élevéé ${ }^{61}$ Ils dépendent dans deux cas du fait que, depuis ce plan de formation, l'entreprise a été certifiée: «du profil d'emploi des salariés »" et « des capacités pour intégrer des exigences qualité ${ }^{6.3}$; les différences de moyennes sont faibles mais elles indiquent une plus grande reconnaissance s'il y a eu certification. Si le répondant estime que le plan de formation a contribué à l'obtention de la certification, nous notons des inégalités de moyennes pour «des capacités à intégrer des objectifs de gestion »" et pour « des capacités pour intégrer des objectifs de production $»^{65}$, le développement apparait alors un peu plus important. Dans trois types de développement, nous trouvons des différences inférables selon que, oui ou non, le plan de formation cofinancé s'inscrivait dans une action de réorganisation du travail: "de la polyvalence " ${ }^{66}$, "des capacités d'analyse des situations de travail ${ }^{67}$ et " des capacités à intégrer des objectifs de gestion " affirmatives correspondent à l'opinion d'une plus grande importance. Enfin, selon que le responsable de l'entreprise considère que l'objectif a été atteint, nous observons des différences de moyennes dans cinq cas: " du profil d'emploi des salariés $»^{69}$, "de la polyvalence $»^{70}$, «des capacités d'analyse des situations de travail $»^{71}$, " des capacités pour intégrer des objectifs de production $»^{72}$ et " des capacités pour intégrer des exigences qualité $»^{73}$; quand on répond de façon affirmative, l'aspect du développement se veut un peu plus important.

Les constats relatifs au développement ne dépendent du critère principal qui a prévalu pour le choix de l'organisme de formation que pour « des

\footnotetext{
${ }^{61} \mathrm{t}_{(161)}=-3,93 ; \mathrm{p}<0,05$.

${ }^{62} \mathrm{t}_{(62,16)}=-2,80 ; \mathrm{p}<0,05$.

${ }_{6.3} t_{(159)}=-2,60 ; p<0,05$.

${ }^{64} \mathrm{t}_{(36)}=-4,30 ; \mathrm{p}<0,05$.

${ }_{65} \mathrm{t}_{(36)}=-2,97 ; \mathrm{p}<0,05$.

${ }^{66} \mathrm{t}_{(163)}=-2,41 ; \mathrm{p}<0,05$.

${ }^{67} \mathrm{t}_{(161)}=-2,03 ; \mathrm{p}<0,05$.

${ }^{68} t_{(159)}=-3,01 ; p<0,05$.

6) $\mathrm{t}_{(110)}=-2,98 ; \mathrm{p}<0,05$.

${ }^{70} \mathrm{t}_{(117)}=-2,49 ; \mathrm{p}<0,05$.

${ }^{71} \mathrm{t}_{(115)}=-2,12 ; \mathrm{p}<0,05$.

${ }_{72} t_{(114)}=-3,25 ; \mathrm{p}<0,05$.

${ }^{73} \mathrm{t}_{(41,33)}=-4,56 ; \mathrm{p}<0,05$.
} 
capacités pour intégrer des exigences qualité »; on y accorde une plus grande importance quand il s'est agi d'une antériorité de prestation, de recommandations de l'OPCA ou de celles d'entreprises partenaires; ce type de développement apparaît moins important quand le choix a été défini par la fédération professionnelle, par le groupe ou par les donneurs d'ordres ${ }^{74}$. Cela semble indiquer que, suite à la formation, l'entreprise a davantage de chance de voir se réaliser un développement des capacités des salariés à intégrer des exigences qualité quand cette formation a vu le jour au sein du réseau expérientiel de l'entreprise (antériorité, recommandation, partenariat).

$\mathrm{Si}$ un cahier des charges a été rédigé, la moyenne est légèrement plus élevée que dans le cas contraire ${ }^{75}$; cette rédaction n'a d'incidence sur l'appréciation d'aucun autre aspect du développement. Le cahier des charges, nous l'avons vu, ne garantit pas une augmentation des chances de voir le développement des capacités des salariés s'accroître. Le questionnaire, nous le savons, permet de savoir si la proposition de formation a été «conçue et réalisée par vous » ou si elle est « repérable dans l'offre existante de l'organisme »; cette alternative donne lieu à deux inégalités des moyennes: l'une pour " des capacités de communication ${ }^{76}$ et l'autre pour " des capacités pour intégrer des objectifs de production ${ }^{77}$; l'importance parait alors un peu plus grande si elle a été produite par l'entreprise elle-même. Le fait que l'organisme de formation soit ou non « intervenu en amont dans le montage du dossier » n'a pas d'influence sur les positions que suscitent les divers composants du développement. Trois questions, on s'en souviendra, vont un peu dans le même sens: "dans tous les cas, pensez-vous que vos objectifs et vos attentes ont été compris? ", "vos objectifs et vos attentes ont-ils été pris en compte? » et «les actions de formation qui vous ont été proposées étaient-elles adaptés? ». Nous avons déjà vu que les réponses se répartissent dans deux catégories « complètement » et «partiellement». En vertu des deux premières questions, les différences ne sont significatives que pour un seul type de développement, et dans les deux cas: « des capacités pour intégrer des exigences qualité $)^{78}$; si la réponse est «complètement », la moyenne montre qu'on y accorde un peu plus d'importance. En fonction de la troisième, nous ne lisons que des égalités de moyennes. Si les responsables des entreprises affirment qu'ils procéderaient de la même

\footnotetext{
${ }^{74}$ Test Kruskal-Wallis: $\chi_{(6)}^{2}=13,64 ; \mathrm{p}<0,05$.

${ }^{75} \mathrm{t}_{(153)}=-3,54 ; \mathrm{p}<0,05$.

${ }^{76} \mathrm{t}_{(145)}=-2,59 ; \mathrm{p}<0,05$.

${ }^{77} \mathbf{t}_{(144)}=-2,57 ; \mathrm{p}<0,05$.

${ }^{78} \mathrm{t}_{(56,56)}=-2,43 ; \mathrm{p}<0,05$ et $\mathrm{t}_{(53,55)}=-2,61 ; \mathrm{p}<0,05$.
} 
manière « si c'était à refaire ", leur moyenne est légèrement supérieure que dans le cas opposé pour trois des aspects du développement: «des capacités


communication ${ }^{81}$.

\section{Perception de l'apport de la contribution du FSE et secteur d'activité}

L'opinion que les répondants se font de l'apport du FSE dépend dans certains cas du secteur à l'intérieur duquel opère l'entreprise. S'il est question d'accélération de l'obtention d'une certification qualité, nous notons un plus grand accord dans la construction et un moins grand dans les services aux entreprises ${ }^{82}$. S'il s'agit d'accroissement des gains de productivité, alors c'est dans l'industrie manufacturière, dans la construction et dans le commerce qu'on trouve le plus grand accord, en santé et en action sociale le plus grand désaccord ${ }^{83}$. Si l'on se réfère au maintien sur les marchés existants, alors la santé présente à nouveau le plus grand désaccord ${ }^{84}$. Si l'on songe à une possibilité accrue d'accéder à des formations qualifiantes, le plus grand désaccord, cette fois, se trouve en commerce et le plus grand accord, en santé et en action sociale ${ }^{85}$. Pour les seize autres apports, il n'y a aucune différence significative entre les secteurs.

Ces résultats sont à mettre en lumière du fait que la contribution du FSE était au départ ciblée sur des entreprises dans des secteurs subissant ou anticipant la mutation industrielle au sens restreint du terme. Chemin faisant, et plus particulièrement en région Midi-Pyrénées, une extension de la notion de mutation industrielle s'est opérationnalisée dans le cofinancement d'actions de formation hors du champ initialement prévu. Ainsi, le secteur de la santé/ action sociale comme, par exemple, l'aide aux personnes, a été doté de financements pour des actions qui touchaient à la transformation des métiers et, au risque d'exclusion, des salariés les moins qualifiés. La certification au sens de la normalisation qualité est quasiabsente de ce secteur, la question des gains de productivité ne se pose pas

\footnotetext{
${ }^{79} \mathrm{t}_{(18,09)}=-2,77 ; \mathrm{p}<0,05$.

${ }^{80} \mathrm{t}_{(157)}=-2,25 ; \mathrm{p}<0,05$.

${ }^{81} \mathrm{t}_{(159)}=-1,98 ; \mathrm{p}<0,05$.

${ }^{82}$ Test Kruskal-Wallis: $\chi_{(5)}^{2}=12,38 ; \mathrm{p}<0,05$.

${ }^{83}$ Test Kruskal-Wallis: $\chi_{(5)}^{2}=33,84 ; \mathrm{p}<0,05$.

${ }^{84}$ Test Kruskal-Wallis: $\chi^{2}{ }_{(5)}=11,62 ; \mathrm{p}<0,05$.

${ }^{85}$ Test Kruskal-Wallis: $\chi_{(5)}^{2}=13,60 ; \mathrm{p}<0,05$.
} 
en ces termes pour ces professionnels alors que le besoin de reconnaissance de leur professionnalité par la qualification est primordial.

\section{Perception du changement et secteur d'activité}

Si le secteur d'activité influe parfois sur la manière dont on comprend l'apport du FSE, il intervient fréquemment sur l'opinion qu'on se fait à l'égard du changement que connaissent les entreprises. En fait, il fait varier les opinions sur treize des vingt-deux caractéristiques du changement. Dans onze de ces treize lieux de variation, c'est dans le secteur de la santé et de l'action sociale où l'on trouve que la caractéristique est la moins importante pour définir le changement actuel dans les entreprises. Il s'agit des aspects qui concernent la mondialisation ${ }^{86}$, les nouveaux produits ${ }^{87}$, la fonction commerciale $^{88}$, les donneurs d'ordre ${ }^{89}$, les sous-traitants ${ }^{90}$, la concurrence internationale ${ }^{91}$, l'évolution technologique ${ }^{92}$, la réorganisation du travail ${ }^{93}$, les techniques de fabrication ${ }^{94}$, l'aménagement du temps de travail ${ }^{95}$ et le vieillissement de la population des salariés ${ }^{96}$. Dans presque tous ces cas, c'est le secteur du commerce qui présente les scores qui témoignent de la plus grande préoccupation.

\section{Constats du développement suite aux formations des salariés et secteur d'activité}

Les constats de développement qui ont fait suite à la formation des salariés ne se distinguent que dans deux cas en fonction du secteur dans lequel opère l'entreprise. Il semble, en effet, qu'un développement dans le sens de la polyvalence soit moins manifeste dans le secteur de la construction qu'il l'est dans la restauration, les transports et la communication, d'une part, et

\footnotetext{
${ }^{86}$ Test Kruskal-Wallis: $\chi_{(5)}^{2}=68,94 ; \mathrm{p}<0,05$.

${ }^{87}$ Test Kruskal-Wallis: $\chi_{(5)}^{2}=22,48 ; \mathrm{p}<0,05$.

${ }^{88}$ Test Kruskal-Wallis: $\chi_{(5)}^{2}=40,62 ; \mathrm{p}<0,05$.

${ }^{89}$ Test Kruskal-Wallis: $\chi_{(5)}^{2}=15,38 ; \mathrm{p}<0,05$.

${ }^{90}$ Test Kruskal-Wallis: $\chi^{2}{ }_{(5)}=26,16 ; \mathrm{p}<0,05$.

${ }^{91}$ Test Kruskal-Wallis: $\chi_{(5)}^{2}=66,68 ; \mathrm{p}<0,05$.

92 Test Kruskal-Wallis: $\chi_{(5)}^{2}=39,23 ; \mathrm{p}<0,05$.

${ }^{93}$ Test Kruskal-Wallis: $\chi_{(5)}^{2}=32,48 ; \mathrm{p}<0,05$.

${ }^{94}$ Test Kruskal-Wallis: $\chi^{2}{ }_{(5)}=38,03 ; \mathrm{p}<0,05$.

${ }^{95}$ Test Kruskal-Wallis: $\chi_{(5)}^{2}=15,50 ; \mathrm{p}<0,05$.

${ }^{96}$ Test Kruskal-Wallis: $\chi^{2}{ }_{(5)}=20,60 ; \mathrm{p}<0,05$.
} 
l'industrie manufacturière, d'autre part ${ }^{97}$. Il semble aussi qu'un développement qui consiste en des capacités pour intégrer des objectifs de production soit moins net en santé et en action sociale que dans l'industrie manufacturière ${ }^{98}$.

\section{Une logique systémique}

La manière dont les responsables se représentent leur entreprise témoigne d'une vision plus ou moins structurée, plus ou moins intégrée. Nous y trouvons les signes de perspectives à la fois communes et particulières.

\subsection{Motifs de la demande auprès du FSE et caractéristiques du changement}

La conception du changement auquel est soumise l'entreprise, aujourd'hui, dépend en partie de ce qui a incité à faire demande auprès du FSE, et donc de la manière dont on perçoit les besoins en formation.

Les moyennes pour dix des vingt-deux caractéristiques du changement subissent l'influence de l'importance qu'on accorde à « l'arrivée de concurrents européens ou internationaux ». Quand la concurrence apparait comme " événement prépondérant " parmi les raisons qui poussent à monter un dossier pour le FSE, les dix types de changements, aux yeux du répondant, correspondent davantage à la situation de l'entreprise que lorsqu'elle n'a " pas de relation ». Les différences de moyennes vont toujours dans le même sens. Ainsi, quand la concurrence se veut fortement incitative, le changement est marqué par la mondialisation des échanges économiques $^{99}$, la concurrence internationale ${ }^{100}$ ou la fonction commerciale $^{101}$, par des incertitudes liées au lancement de nouveaux produits $^{102}$ ou les exigences de la clientèle ${ }^{103}$, par l'évolution technologique des machines ${ }^{104}$ ou la transformation des techniques de fabrication ${ }^{105}$, par

\footnotetext{
${ }^{97}$ Test Kruskal-Wallis: $\chi_{(5)}^{2}=12,58 ; \mathrm{p}<0,05$.

${ }^{98}$ Test Kruskal-Wallis: $\chi_{(5)}^{2}=36,70 ; \mathrm{p}<0,05$.

${ }^{99} \mathrm{~F}_{(3 ; 165)}=7,87 ; \mathrm{p}<0,05$.

${ }^{100} \mathbf{F}_{(3 ; 165)}=21,06 ; \mathrm{p}<0,05$

${ }^{101} \mathbf{F}_{(3 ; 162)}=4,00 ; \mathrm{p}<0,05$.

${ }^{102} \mathrm{~F}_{(3 ; 165)}=4,57 ; \mathrm{p}<0,05$.

${ }^{103} \mathrm{~F}_{(3 ; 160)}=3,86 ; \mathrm{p}<0,05$.

${ }^{104} \mathrm{~F}_{(3 ; 164)}=6,51 ; \mathrm{p}<0,05$.

${ }^{105} \mathrm{~F}_{(3 ; 160)}=5,53 ; \mathrm{P}<0,05$.
} 
des relations renforcées avec les donneurs d'ordres ${ }^{106}$ ou par un changement de direction ${ }^{107}$ et, enfin, par le vieillissement de la population salariée $e^{108}$.

Selon que « l'arrivée d'un nouveau produit sur le marché » constitue un «événement prépondérant », un «événement important», un «événement secondaire » ou un événement sans relation pour la demande auprès du FSE, la perception du changement varie pour huit des vingt-deux caractéristiques: la mondialisation des échanges économiques ${ }^{109}$, la concurrence internationale ${ }^{110}$, la fusion avec une autre entité ${ }^{111}$, la diversification des produits $^{112}$, l'évolution technologique des machines ${ }^{113}$, la réorganisation du travail pour augmenter la productivité ${ }^{114}$, la transformation des techniques de fabrication ${ }^{115}$ et le vieillissement de la population salariée ${ }^{116}$. Les données vont dans le même sens que pour l'arrivée de concurrents: quand il s'agit d'un événement prépondérant, la caractéristique se veut plus associée que lorsqu'on a affaire à un événement sans pertinence.

«L'intégration de nouvelles technologies de production » influe, elle, sur treize caractéristiques: la mondialisation des échanges économiques ${ }^{117}$, la concurrence internationale ${ }^{118}$, l'optimisation de la fonction commerciale ${ }^{119}$, les incertitudes liées au lancement de nouveaux produits ${ }^{120}$, la nécessaire diversification des produits ${ }^{121}$, les changements des exigences de la clientèle ${ }^{122}$, l'évolution technologique des machines ${ }^{123}$, la transformation des techniques de fabrication ${ }^{124}$, la mise en place d'un système informatique en

\footnotetext{
${ }^{1066} \mathrm{~F}_{(3 ; 164)}=3,04 ; \mathrm{p}<0,05$.

${ }^{107} \mathrm{~F}_{(3 ; 163)}=3,34 ; \mathrm{p}<0,05$.

${ }^{108} \mathrm{~F}_{(3 ; 160)}=3,99 ; \mathrm{P}<0,05$.

${ }^{109} \mathrm{~F}_{(3 ; 164)}=6,50 ; \mathrm{p}<0,05$.

${ }^{110} \mathrm{~F}_{(3 ; 164)}=6,13 ; \mathrm{p}<0,05$.

${ }^{111} \mathrm{~F}_{(3 ; 163)}=3,82 ; \mathrm{p}<0,05$.

${ }^{112} \mathrm{~F}_{(3 ; 165)}=3,63 ; \mathrm{p}<0,05$.

${ }^{113} \mathrm{~F}_{(3 ; 163)}=3,17 ; \mathrm{p}<0,05$.

${ }^{114} \mathrm{~F}_{(3 ; 166)}=3,20 ; \mathrm{p}<0,05$.

${ }^{115} \mathrm{~F}_{(3 ; 160)}=3,44 ; \mathrm{p}<0,05$.

${ }^{116} \mathrm{~F}_{(3 ; 166)}=3,72 ; \mathrm{p}<0,05$.

${ }^{117} \mathrm{~F}_{(3 ; 165)}=16,40 ; \mathrm{p}<0,05$.

${ }^{118} \mathrm{~F}_{(3 ; 165)}=6,02 ; \mathrm{p}<0,05$.

${ }^{119} \mathrm{~F}_{(3 ; 162)}=4,52 ; \mathrm{p}<0,05$.

${ }^{120} \mathrm{~F}_{(3 ; 165)}=5,25 ; \mathrm{p}<0,05$.

${ }^{121} \mathrm{~F}_{(3 ; 166)}=5,19 ; \mathrm{p}<0,05$.

${ }^{122} \mathrm{~F}_{(3 ; 166)}=2,77 ; \mathrm{p}<0,05$.

${ }^{123} \mathrm{~F}_{(3 ; 164)}=17,51 ; \mathrm{p}<0,05$.

${ }^{124} \mathrm{~F}_{(3 ; 160)}=22,38 ; \mathrm{p}<0,05$.
} 
réseau pour l'ensemble des fonctions de l'entreprise ${ }^{125}$, l'aménagement et la réduction du temps de travail ${ }^{126}$, la réorganisation du travail pour augmenter la productivité ${ }^{127}$, les relations renforcées avec les donneurs d'ordres ${ }^{128}$ et avec les sous-traitants ${ }^{129}$. Quand le motif se veut prépondérant, la moyenne révèle une plus grande insistance sur la caractéristique.

Si le motif de la demande au FSE est « la fusion avec une autre entité », alors il n'a d'incidence que si le changement se manifeste comme fusion ${ }^{130}$. Il s'agit d'une relation tautologique. Mais nous ne pouvons que remarquer l'absence de relation de cet incitatif avec les autres aspects du changement.

Quand «l'évolution de l'outil de production » est pris en compte à titre de facteur de la demande pour le concours du FSE, quatorze des vingt-deux caractéristiques du changement connaissent des variations qu'on ne peut attribuer au hasard. Si cette évolution se signale comme événement prépondérant, le changement tend à se distinguer par les aspects suivants: le degré de mondialisation des échanges économiques ${ }^{131}$, l'intensité de la concurrence internationale ${ }^{132}$, l'optimisation de la fonction commerciale ${ }^{133}$, la nécessaire diversification des produits ${ }^{134}$, les changements des exigences de la clientèle ${ }^{135}$, les incertitudes liées au lancement de nouveaux produits ${ }^{136}$, les relations renforcées avec les donneurs d'ordres ${ }^{137}$ et avec les soustraitants ${ }^{138}$, les relations avec les entreprises ayant des activités complémentaires à la production de l'entreprise ${ }^{139}$, la fusion avec une autre ou d'autres entités $^{140}$, l'évolution technologique des machines ${ }^{141}$, la réorganisation du travail pour augmenter la productivité ${ }^{142}$ et la transformation des techniques

$$
\begin{aligned}
& { }^{125} \mathrm{~F}_{(3 ; 167)}=3,03 ; \mathrm{p}<0,05 \text {. } \\
& { }^{126} \mathrm{~F}_{(3 ; 168)}=2,68 ; \mathrm{p}<0,05 \text {. } \\
& { }^{127} \mathrm{~F}_{(3 ; 167)}=10,44 ; \mathrm{p}<0,05 \text {. } \\
& { }^{128} \mathrm{~F}_{(3 ; 164)}=2,81 ; \mathrm{p}<0,05 \text {. } \\
& { }^{129} \mathrm{~F}_{(3 ; 159)}=3,20 ; \mathrm{p}<0,05 \text {. } \\
& { }^{130} \mathrm{~F}_{(3 ; 164)}=6,52 ; \mathrm{p}<0,05 \text {. } \\
& { }^{131} \mathrm{~F}_{(3 ; 163)}=10,14 ; \mathrm{p}<0,05 \text {. } \\
& { }^{132} \mathrm{~F}_{(3 ; 163)}=3,98 ; \mathrm{p}<0,05 \text {. } \\
& { }^{133} \mathrm{~F}_{(3 ; 160)}=4,17 ; \mathrm{p}<0,05 \text {. } \\
& { }^{134} \mathrm{~F}_{(3 ; 164)}=4,58 ; \mathrm{p}<0,05 \text {. } \\
& { }^{135} \mathrm{~F}_{(3 ; 164)}=8,89 ; \mathrm{p}<0,05 \text {. } \\
& { }^{136} \mathrm{~F}_{(3 ; 163)}=7,27 ; \mathrm{p}<0,05 \text {. } \\
& { }^{137} \mathrm{~F}_{(3 ; 162)}=4,33 ; \mathrm{p}<0,05 \text {. } \\
& { }^{138} \mathrm{~F}_{(3 ; 157)}=4,62 ; \mathrm{p}<0,05 \text {. } \\
& { }^{139} \mathrm{~F}_{(3 ; 158)}=4,12 ; \mathrm{p}<0,05 \text {. } \\
& { }^{140} \mathrm{~F}_{(3 ; 161)}=3,54 ; \mathrm{p}<0,05 \text {. } \\
& { }^{141} \mathrm{~F}_{(3 ; 162)}=14,12 ; \mathrm{p}<0,05 \text {. } \\
& { }^{142} \mathrm{~F}_{(3 ; 165)}=6,33 ; \mathrm{p}<0,05 \text {. }
\end{aligned}
$$


de fabrication ${ }^{143}$. Une caractéristique ne répond pas à cette logique, mais elle donne quand même lieu à des différences significatives. En effet, l'aménagement et la réduction du temps de travail ${ }^{144}$ ne constituent un aspect moins caractéristique du changement si l'évolution de l'outil de production représente un événement prépondérant ou un événement sans relation que lorsque cette évolution se présente comme événement important ou secondaire. Cette étrange relation entre les deux variables ne doit cependant pas occulter le modèle qui se dégage de l'ensemble des analyses.

Les répondants sont aussi appelés à se prononcer sur « la définition d'une nouvelle organisation du travail ", dans son rapport avec la demande qui est déposée auprès du FSE. Leur position influe sur sept aspects du changement: l'optimisation de la fonction commerciale ${ }^{145}$, la diversification des produits ${ }^{146}$, l'évolution technologique des machines ${ }^{147}$, la mise en place d'un nouveau système de communication externe ${ }^{148}$, la réorganisation du travail pour augmenter la productivite ${ }^{149}$, la mise en place d'un système qualité $^{150}$ et la gestion prévisionnelle des emplois et des compétences ${ }^{151}$. Si l'organisation du travail est prépondérante en ce qui a trait à la demande, les caractéristiques se signalent par l'importance qu'on leur accorde.

« L'accompagnement d'une nouvelle organisation du travail » agit, lui aussi, sur sept aspects du changement: le degré de mondialisation des échanges économiques ${ }^{152}$, les changements des exigences de la clientèle ${ }^{153}$, l'optimisation de la fonction commerciale ${ }^{154}$, l'évolution technologique des machines ${ }^{155}$, la réorganisation du travail pour augmenter la productivité ${ }^{156}$, la transformation des techniques de fabrication ${ }^{157}$ et l'aménagement et la réduction du temps de travail ${ }^{158}$. Si le responsable de l'entreprise considère

\footnotetext{
${ }^{143} \mathrm{~F}_{(3 ; 158)}=20,76 ; \mathrm{p}<0,05$.

${ }^{144} \mathrm{~F}_{(3 ; 160)}=3,66 ; \mathrm{p}<0,05$.

${ }^{145} \mathrm{~F}_{(3 ; 162)}=5,68 ; \mathrm{p}<0,05$.

${ }^{146} \mathrm{~F}_{(3 ; 166)}=6,57 ; \mathrm{p}<0,05$.

${ }^{147} \mathrm{~F}_{(3 ; 164)}=2,81 ; \mathrm{p}<0,05$.

${ }^{148} \mathrm{~F}_{(3 ; 164)}=3,98 ; \mathrm{p}<0,05$.

${ }^{149} \mathrm{~F}_{(3 ; 167)}=8,10 ; \mathrm{p}<0,05$.

${ }^{150} \mathrm{~F}_{(3 ; 167)}=5,40 ; \mathrm{p}<0,05$.

${ }^{151} \mathrm{~F}_{(3 ; 160)}=2,82 ; \mathrm{p}<0,05$.

${ }^{152} \mathrm{~F}_{(3 ; 167)}=4,61 ; \mathrm{p}<0,05$.

${ }^{153} \mathrm{~F}_{(3 ; 168)}=2,80 ; \mathrm{p}<0,05$.

${ }^{154} \mathrm{~F}_{(3 ; 163)}=2,78 ; \mathrm{p}<0,05$.

${ }^{155} \mathrm{~F}_{(3 ; 160)}=5,24 ; \mathrm{p}<0,05$.

${ }^{156} \mathrm{~F}_{(3 ; 169)}=12,29 ; \mathrm{p}<0,05$.

${ }^{157} \mathrm{~F}_{(3 ; 161)}=5,42 ; \mathrm{p}<0,05$.

${ }^{158} \mathrm{~F}_{(3 ; 170)}=2,86 ; \mathrm{p}<0,05$.
} 
que l'accompagnement est sans relation avec la demande, les caractéristiques se présentent à lui comme moins significatives.

«La baisse d'activité de l'entreprise », quelle que soit son importance, n'est associée à aucune des dimensions du changement.

Six des aspects du changement sont affectés par la manière dont on conçoit le rôle des «nouvelles exigences du donneur d'ordre »: les changements des exigences de la clientèle ${ }^{159}$, les relations renforcées avec les donneurs d'ordres ${ }^{160}$ (association tautologique), les relations avec les soustraitants ${ }^{161}$, les relations avec les entreprises ayant des activités complémentaires à la production de l'entreprise ${ }^{162}$, la fusion avec une autre entité ${ }^{163}$, la mise en place d'un système qualité ${ }^{164}$. "De nouvelles exigences des clients » différencient les moyennes dans trois cas: les changements des exigences de la clientèle ${ }^{165}$, la nécessaire diversification des produits ${ }^{166}$ et l'évaluation nécessaire des compétences des salariés ${ }^{167}$. Trois dimensions du changement varient en fonction de l'impact que « la volonté de mettre en place un système qualité » (association tautologique) a eu sur la décision de mettre sur pied une demande: les relations renforcées avec les donneurs d'ordres ${ }^{168}$, la fusion avec une autre ou d'autres entités ${ }^{169}$ et la mise en place d'un système qualité ${ }^{170}$. Le fait que la « diversification de l'activité » motive la demande à quelque degré ou non distingue les positions pour sept aspects du changement: les changements des exigences de la clientèle ${ }^{171}$, l'optimisation de la fonction commerciale ${ }^{172}$, les relations renforcées avec les donneurs d'ordres ${ }^{173}$, les relations avec les entreprises ayant des activités complémentaires à la production de l'entreprise ${ }^{174}$, la nécessaire diversification des

\footnotetext{
${ }^{159} \mathrm{~F}_{(3 ; 161)}=3,88 ; \mathrm{p}<0,05$.

${ }^{160} \mathrm{~F}_{(3 ; 159)}=17,14 ; \mathrm{p}<0,05$

${ }^{161} \mathrm{~F}_{(3 ; 154)}=6,05 ; \mathrm{p}<0,05$.

${ }^{162} \mathrm{~F}_{(3,155)}=2,89 ; \mathrm{p}<0,05$.

${ }^{163} \mathrm{~F}_{(3 ; 159)}=2,69 ; \mathrm{p}<0,05$.

${ }^{164} \mathrm{~F}_{(3,162)}=2,82 ; \mathrm{p}<0,05$

${ }^{165} \mathrm{~F}_{(3,167)}=7,32 ; \mathrm{p}<0,05$.

${ }^{166} \mathrm{~F}_{(3 ; 167)}=3,42 ; \mathrm{p}<0,05$.

${ }^{167} \mathrm{~F}_{(3 ; 167)}=2,98 ; \mathrm{p}<0,05$.

${ }^{168} \mathrm{~F}_{(3 ; 165)}=3,85 ; \mathrm{p}<0,05$.

${ }^{169} \mathrm{~F}_{(3 ; 164)}=2,90 ; \mathrm{p}<0,05$.

${ }^{170} \mathrm{~F}_{(3 ; 168)}=14,86 ; \mathrm{p}<0,05$.

${ }^{171} \mathrm{~F}_{(3 ; 166)}=3,56 ; \mathrm{p}<0,05$.

${ }^{172} \mathrm{~F}_{(3 ; 162)}=4,46 ; \mathrm{p}<0,05$.

${ }^{173} \mathrm{~F}_{(3 ; 165)}=4,73 ; \mathrm{p}<0,05$.

${ }^{174} \mathrm{~F}_{(3 ; 160)}=6,22 ; \mathrm{p}<0,05$.
} 
produits $^{175}$, la réorganisation du travail pour augmenter la productivité $e^{176}$ et la mise en place d'un système qualité ${ }^{177}$. Pour chacune de ces quatre séries d'analyses, la variation des moyennes va dans le même sens et elle correspond à la logique qui domine dans les résultats qui précèdent.

Il y a un dernier incitatif: « le changement de direction ». Il intervient de façon significative dans quatre cas: le haut degré de mondialisation des échanges économiques ${ }^{178}$, la nécessaire diversification des produits ${ }^{179}$, le changement de direction ${ }^{180}$ (relation tautologique) et le plan social ${ }^{181}$. Cette fois, cependant, les différences de moyennes ne répondent pas toutes à la même logique: elles le font pour la diversification des produits et pour le changement de direction; elles ne le font pas pour les techniques de fabrication et pour le plan social.

On peut observer l'existence d'un certain lien entre la façon dont les responsables des entreprises se représentent le changement, aujourd'hui, et celle dont ils motivent la demande qu'ils ont déposée auprès du FSE. Ce lien, dans la presque totalité des cas, est consistant: si le motif se veut déterminant, la caractéristique du changement apparait comme importante, comme plus importante, en tout cas, que si sont évoquées les autres possibilités. Ce lien, toutefois, n'est pas parfait. Il renvoie bien à une tendance, mais non pas à des positions identiques. Il est tout à fait possible que la demande ait été motivée de façon prépondérante par tel événement et que la manière dont se caractérise le changement pour un aspect correspondant fasse état d'un jugement plus nuancé. Nous le voyons même pour les relations tautologiques. Ce lien n'est pas tout à fait symétrique et il est généralement pluriel: un même incitatif se rattache souvent à plusieurs caractéristiques du changement. En cela, nous pouvons constater que les responsables d'entreprises sont animés par des visions composites et, à maints égards, particulières. Mais il faut aussi constater que bon nombre de dimensions du changement, en vertu de chacun des motifs, ne connaissent aucune variation; jamais plus de quatorze caractéristiques sur vingt-deux ne présentent des moyennes inégales. Cela montre bien que les responsables des entreprises agissent aussi dans un environnement qui leur renvoie une

\footnotetext{
${ }^{175} \mathrm{~F}_{(3 ; 166)}=14,49 ; \mathrm{p}<0,05$.

${ }^{176} \mathrm{~F}_{(3 ; 167)}=2,82 ; \mathrm{p}<0,05$

${ }^{177} \mathrm{~F}_{(3 ; 167)}=5,02 ; \mathrm{p}<0,05$.

${ }^{178} \mathrm{~F}_{(3 ; 1,165)}=2,98 ; \mathrm{p}<0,05$.

${ }^{179} \mathrm{~F}_{(3 ; 166)}=2,95 ; \mathrm{p}<0,05$.

${ }^{180} \mathrm{~F}_{(3 ; 163)}=4,03 ; \mathrm{p}<0,05$.

${ }^{181} \mathrm{~F}_{(3 ; 161)}=3,89 ; \mathrm{p}<0,05$.
} 
image semblable. Des aspects du changement se manifestent plus communément que les autres: la nécessaire diversification des produits, les changements des exigences de la clientèle (sept fois chacun); le degré de mondialisation, l'optimisation de la fonction commerciale, les relations renforcées avec les donneurs d'ordres, l'évolution technologique des machines, la réorganisation du travail pour augmenter la productivité, la transformation des techniques de fabrication (six fois chacun). Nous voyons bien là que, lorsque des dimensions du changement donnent lieu à des visions différenciées, c'est pour distinguer des autres les entreprises qui sont fortement déterminées par les exigences du libéralisme économique et d'une économie mondialisée.

\subsection{Motifs de la demande auprès du FSE et clefs de la réussite d'une entreprise}

On peut aborder le rapport entre les motifs de la demande au FSE et la manière dont sont comprises « les clefs de la réussite d'une entreprise » tout comme nous l'avons fait pour l'interprétation du changement: en comparant les moyennes. La comparaison est alors effectuée en fonction des catégories de réponses relatives à chacun des incitatifs pour la position qui correspond à chacune des clefs que suggère le questionnaire. Les moyennes montrent normalement que les clefs sont importantes aux yeux des répondants. Mais il y a tout de même quelques lieux de variation.

En reprenant les motifs dans le même ordre, nous découvrons que la façon de concevoir le rôle de "l'arrivée de concurrents européens ou internationaux » dans la demande n'agit que sur une seule des treize clefs: avoir un outil de production adapté ${ }^{182}$. La différence est faible, mais elle montre que si le motif a été prépondérant, le répondant est davantage d'accord pour affirmer que ces outils de production adaptés constituent une clef du succès.

L'opinion sur deux des treize clefs varie selon l'importance qu'on accorde à «l'arrivée d'un produit sur notre marché »: mener des activités internes de recherche ${ }^{183}$ et être réactif à son environnement ${ }^{184}$. Le degré d'assentiment sur l'importance de ces clefs est légèrement plus marqué quand le responsable de l'entreprise est convaincu du fait que l'arrivée des produits a motivé la demande.

\footnotetext{
${ }^{182} \mathrm{~F}_{(3 ; 171\}}=3,51 ; \mathrm{p}<0,05$.

${ }^{183} \mathrm{~F}_{(3 ; 169)}=3,04 ; \mathrm{p}<0,05$.

${ }^{184} \mathrm{~F}_{(3 ; 170)}=3,10 ; \mathrm{p}<0,05$.
} 
«L'intégration de nouvelles technologies », comme incitatif de la demande, fait varier les moyennes pour six clefs: mener des activités internes de recherche ${ }^{185}$, avoir du personnel à la pointe de la compétence $e^{186}$, développer le dialogue social au sein de l'entreprise ${ }^{187}$, avoir un outil de production adaptét ${ }^{188}$, avoir un système informatique reliant toutes les fonctions de l'entreprise ${ }^{189}$ et avoir une entreprise avec une organisation du travail « optimisée ${ }^{190}$. La moyenne est légèrement plus élevée quand le motif semble prépondérant.

«La fusion avec une autre entité » est sans effet sur l'opinion relative aux diverses clefs.

«L'évolution de l'outil de production » provoque des différences de moyennes pour deux clefs: mener des activités internes de recherche ${ }^{191}$ et avoir un outil de production adapté ${ }^{192}$. Si le motif apparaît sans relation, nous inclinons moins à penser que la clef est déterminant.

L'opinion varie sur l'importance de deux clefs quand intervient la position sur « la définition d'une nouvelle stratégie ». L'une d'elle, « avoir une entreprise avec une organisation du travail optimisée $»{ }^{193}$, s'interprète dans le sens d'une prépondérance du motif qui correspond à une plus grande importance de la clef. L'autre, « avoir du personnel à la pointe de la compétence ${ }^{194}$, présente des moyennes qui vont dans tous les sens: la valeur qu'il faut accorder à la clef est au plus faible quand la nouvelle stratégie représente un événement important, au plus fort, quand l'événement est considéré comme prépondérant; elle est presque équivalente à celle-ci quand l'événement est catégorisé comme étant sans relation.

L'opinion sur deux clefs est affectée par le motif qu'est «l'accompagnement d'une nouvelle organisation du travail $»$ : avoir un outil de production adapté $^{195}$ et avoir une entreprise avec une organisation du travail optimi-

\footnotetext{
${ }^{185} \mathrm{~F}_{(3 ; 170)}=4,36 ; \mathrm{p}<0,05$.

${ }^{186} \mathrm{~F}_{(3 ; 171)}=3,06 ; \mathrm{p}<0,05$.

${ }^{187} \mathrm{~F}_{(3 ; 171)}=3,94 ; \mathrm{p}<0,05$.

${ }^{188} \mathrm{~F}_{(3 ; 170)}=4,98 ; \mathrm{p}<0,05$.

${ }^{189} \mathrm{~F}_{(3 ; 171)}=5,39 ; \mathrm{p}<0,05$.

${ }^{1 \times)} \mathrm{F}_{(3 ; 171)}=3,99 ; \mathrm{p}<0,05$.

${ }^{191} \mathrm{~F}_{(3 ; 168)}=3,99 ; \mathrm{p}<0,05$.

${ }^{192} \mathrm{~F}_{(3 ; 168)}=7,38 ; \mathrm{p}<0,05$.

${ }^{193} \mathrm{~F}_{(3 ; 171)}=4,02 ; \mathrm{p}<0,05$.

${ }^{194} \mathrm{~F}_{(3 ; 171)}=3,03 ; \mathrm{p}<0,05$.

${ }^{195} \mathrm{~F}_{(3 ; 171)}=4,30 ; \mathrm{p}<0,05$.
} 
sée $^{196}$. Si le motif apparaît comme prépondérant, l'importance de la clef est plus marquée.

"La baisse d'activité de l'entreprise », à titre de déterminant de la demande, agit sur l'opinion relative à une clef: être à l'écoute de son environnement ${ }^{197}$. L'inégalité des moyennes ne suit pas une logique particulière.

$\mathrm{Si}$ « de nouvelles exigences du donneur d'ordre » représentent un motif prépondérant de la demande, alors les répondants accordent un peu plus d'importance à ces deux clefs: diversifier ses activités ${ }^{198}$ et avoir un outil de production adapté ${ }^{199}$. Il en va de même de l'influence de l'incitatif « de nouvelles exigences des clients $»$; la question de la diversification des activités $^{200}$ surgit à nouveau, mais, cette fois, elle est accompagnée de celle qui a trait à l'optimisation du travail ${ }^{201}$.

L'opinion est sensible à l'influence de « la volonté de mettre en place un système qualité " dans trois cas: diversifier ses activités ${ }^{202}$, mener des activités internes de recherche ${ }^{203}$ et considérer le personnel comme une ressource au service de la stratégie $e^{204}$. Si le motif est événement prépondérant ou s'il est sans relation, on accorde plus d'importance à la clef?

"Une diversification de l'activité » correspond à une diversification des activités $^{205}$ : il va sans dire que s'il s'agit d'un motif prépondérant, il s'agit également d'une clef importante.

"Le changement de direction », s'il constitue un motif décisif, intensifie quelque peu l'importance à accorder au fait d'avoir du personnel compétent ${ }^{206}$.

La plupart de ces analyses de variance indiquent, par le peu de clefs qui subissent l'influence des motifs, qu'on a affaire à une grande entente entre les répondants, une entente qui va dans le sens de la reconnaissance de l'importance à accorder, en principe, à chacune des clefs de la réussite. L'ensemble des motifs ne fait varier les opinions que sur une moyenne de

\footnotetext{
${ }^{196} \mathrm{~F}_{(3 ; 173)}=8,10 ; \mathrm{p}<0,05$.

${ }^{197} \mathrm{~F}_{(3 ; 169)}=2,93 ; \mathrm{p}<0,05$.

${ }^{198} \mathrm{~F}_{(3 ; 163)}=2,87 ; \mathrm{p}<0,05$.

${ }^{199} \mathrm{~F}_{(3 ; 164)}=3,43 ; \mathrm{p}<0,05$.

${ }^{200} \mathrm{~F}_{(3,170)}=3,19 ; \mathrm{p}<0,05$.

${ }^{201} \mathrm{~F}_{(3: 172)}=3,62 ; \mathrm{p}<0,05$.

${ }^{202} \mathrm{~F}_{(3,170)}=5,91 ; \mathrm{p}<0,05$.

${ }^{203} \mathrm{~F}_{(3 ; 170)}=5,91 ; \mathrm{p}<0,05$.

${ }^{204} \mathrm{~F}_{(3 ; 172)}=2,75 ; \mathrm{p}<0,05$.

${ }^{205} \mathrm{~F}_{(3 ; 169)}=6,25 ; \mathrm{p}<0,05$.

${ }^{206} \mathrm{~F}_{(3 ; 171)}=4,78 ; \mathrm{p}<0,05$
} 
1,92 clef, c'est-à-dire que, sur les treize clefs suggérées, il s'en dégage, en moyenne, moins de deux. Il y a toutefois un cas d'exception, celui qui a trait à l'influence de l'intégration des nouvelles technologies, où six clefs connaissent des variations d'opinion. Lorsque cette intégration préoccupe, cela accentue le questionnement sur plusieurs dimensions de l'entreprise: les activités de recherche, la compétence du personnel, le dialogue au sein de l'entreprise, les outils de production, l'informatique, l'organisation du travail. Il est, par ailleurs, des clefs qui se manifestent avec plus de récurrence: avoir des outils de production adaptés (cinq fois), diversifier ses activités, mener des activités internes de recherche et avoir une entreprise avec une organisation du travail optimisée (quatre fois chacune). Elles correspondent difficilement à une seule symbolique.

\subsection{Perception du changement et clefs de la réussite d'une entreprise}

En principe, il devrait y avoir un lien, dans l'esprit des responsables d'entreprise, entre la manière dont on conçoit le changement aujourd'hui et la façon dont on se représente les clefs de la réussite d'une entreprise. Pour le mesurer, l'opinion sur chacune des clefs est mise en relation avec les vingt-deux énoncés relatifs au changement.

Pas moins de dix-huit des vingt-deux aspects du changement parviennent à s'inscrire dans les équations de régression. Cela témoigne de la pluralité des informations que manipulent les responsables d'entreprise, de la difficulté à réduire cette information. Le caractère pluriel de leur vision se révèle aussi dans le fait que la plupart des équations comprennent plus d'une des caractéristiques du changement. Toutes ces caractéristiques, toutefois, n'occupent que le même espace dans leur esprit. Neuf d'entre eux ne surgissent qu'une seule fois. Le problème de la diversification des produits se manifeste quatre fois. Six aspects se présentent trois fois; ils ont trait soit au personnel, soit à la circulation de l'information, soit à la clientèle. Nous pouvons ainsi observer une certaine structure où domine, dans l'ensemble, le rapport personnel-informationproduit-clientèle. Cette structure est importante, mais elle n'est pas réductible; elle n'élimine pas les autres préoccupations. Même dans la relation des caractéristiques du changement avec les clefs du changement, la variance expliquée n'atteint qu'exceptionnellement la valeur de $30 \%$. C'est donc dire que plusieurs autres facteurs entrent en ligne de compte. Cette faiblesse de l'influence, même si elle est loin d'être négligeable, indique en même temps que les considérations sur les clefs du succès ne varient pas simplement en fonction 
de la manière dont on perçoit le changement. La détermination est réelle, sans être complète. Mais, au-delà de cette incomplétude, il faut voir également que, à maints égards, plusieurs notions s'imposent aux responsables des entreprises de façon transcendante de telle sorte que leurs positions se ressemblent, donc, ne varient pas en fonction d'autre chose parce qu'elle ont beaucoup en commun. Plus est faible une variation, moins elle est explicable en fonction d'autre chose. Nous entrevoyons ici un complexe dans lequel se révèlent deux tendances contradictoires: homogénéité et différenciation des positions, intégration et non-intégration des composants.

\section{Tableau 4}

Régression multiple de toutes les variables relatives à la représentation actuelle du changement

( $1=$ de manière incontoumable et $4=$ de manière insignifiante)

dont la contribution est apparue significative pour les opinions sur les clefs de la réussite

d'une entreprise ( $1=$ tout à fait d'accord et $4=$ pas du tout d'accord $)$.

Coefficient standardisé $(\boldsymbol{\beta})$ et variance expliquée

\begin{tabular}{|c|c|c|c|}
\hline Clefs de la réussite d'une entreprise & $\begin{array}{l}\text { Variables relatives à la représentation } \\
\text { actuelle du changement }\end{array}$ & $\beta$ & $\mathrm{R}^{2}$ \\
\hline Se centrer sur son métier, son savoir faire. & $\begin{array}{l}\text { Vieillissement de la population des salarićs } \\
\text { Gestion des emplois et des compétences }\end{array}$ & $\begin{array}{r}0,21 \\
-0,20\end{array}$ & 0 \\
\hline Diversifier ses activités & Diversification des produits. & 0 & 0 \\
\hline Étre à l'écoute de son environnement . . . . . . & Élévation des competences des salariés .... & 0 & 0 \\
\hline Mener des activités internes de recherche $\ldots . .$. & $\begin{array}{l}\text { Diversification des produits .............. } \\
\text { Vieilissement de la population des salariés }\end{array}$ & $\begin{array}{l}0,31 \\
0,19\end{array}$ & 0 \\
\hline Ėtre réactuf à son environnement $\ldots \ldots \ldots \ldots \ldots$ & $\begin{array}{l}\text { Élévation des compétences des salariés ..... } \\
\text { Concurrence internationale } \\
\text { Evolution technologique } \\
\text { Vieillissement de la population des salariés } \\
\text { Diversification des produits } \\
\text { Système qualite }\end{array}$ & $\begin{array}{r}0,36 \\
0,40 \\
-0,27 \\
-0,20 \\
0,25 \\
-0,17\end{array}$ & 0 \\
\hline $\begin{array}{l}\text { Avoir du personnel à la pointe } \ldots \ldots \ldots \ldots \ldots \ldots \\
\text { de la compétence }\end{array}$ & $\begin{array}{l}\text { Mondialisation } \ldots \ldots \ldots \ldots \ldots \ldots \ldots \ldots \\
\text { Diversification des produits }\end{array}$ & $\begin{array}{l}0,23 \\
0,21\end{array}$ & 0 \\
\hline $\begin{array}{l}\text { Combiner qualité des produits et qualité } \\
\text { des ressources humaines }\end{array}$ & $\begin{array}{l}\text { Gestion des emplois et des compétences .... } \\
\text { Clientèle } \\
\text { Changement de direction }\end{array}$ & $\begin{array}{l}0,30 \\
0,27 \\
0,18\end{array}$ & 0 \\
\hline $\begin{array}{l}\text { Développer le dialogue social au sein } \ldots \ldots \ldots \ldots \\
\text { de l'entreprise }\end{array}$ & $\begin{array}{l}\text { Gestion des cmplois et des compétences .... } \\
\text { Systeme de communication } \\
\text { Système informatique } \\
\text { Changement de direction }\end{array}$ & $\begin{array}{r}0,32 \\
0,30 \\
-0,24 \\
0,19\end{array}$ & 0 \\
\hline Avoir un outil de production adaptć & $\begin{array}{l}\text { Clientèle } \ldots \ldots \ldots \ldots \ldots \ldots \ldots \ldots \ldots \\
\text { Sous-traitants } \\
\text { Techniques de fabrication }\end{array}$ & $\begin{array}{l}0,26 \\
0,19 \\
0,18\end{array}$ & 0 \\
\hline $\begin{array}{l}\text { Avoir un système informatique reliant toutes ..... } \\
\text { les fonctions de l'entreprise }\end{array}$ & $\begin{array}{l}\text { Systeme informatique } \ldots \ldots \ldots \ldots \ldots \ldots \ldots \\
\text { Élévation des compétences des salariés } \\
\text { Aménagement du temps de travail }\end{array}$ & $\begin{array}{r}0,36 \\
0,29 \\
-0,17\end{array}$ & 0 \\
\hline $\begin{array}{l}\text { Avoir une entreprise avec une organisation. } \\
\text { du travail optimisée }\end{array}$ & $\begin{array}{l}\text { Donneurs d'ordres .............. } \\
\text { Réotganisation du travail } \\
\text { Systeme de communication }\end{array}$ & $\begin{array}{l}0,25 \\
0,18 \\
0,16\end{array}$ & 0 \\
\hline $\begin{array}{l}\text { Considérer le personnel comme une ressource .... } \\
\text { au service de la stratégie }\end{array}$ & $\begin{array}{l}\text { Clientèle } \ldots \ldots \ldots \ldots \ldots \ldots \ldots \ldots \ldots \\
\text { Donneurs d'ordres }\end{array}$ & $\begin{array}{l}0,19 \\
0,18\end{array}$ & 0 \\
\hline $\begin{array}{l}\text { Tenir compre des compétences du personnel .... } \\
\text { pour définir la stratégie }\end{array}$ & $\begin{array}{l}\text { Plan social } \ldots \ldots \ldots \ldots \ldots \ldots \ldots \ldots \ldots \\
\text { Système de communication } \\
\text { Système informatique }\end{array}$ & $\begin{array}{r}0,26 \\
0,26 \\
-0,25\end{array}$ & 0 \\
\hline
\end{tabular}




\subsection{Perception du changement et plan d'action associé à une stratégie}

Dans le questionnaire, une question demande " votre entreprise a-t-elle une stratégie?». Une question subséquente est ainsi formulée: « cette stratégie est-elle associée à un ou des plans d'action? » Dans le cas de réponses affirmatives, les répondants sont invités à signaler, pour divers plans d'action - seize au total-, le niveau de programmation. Deux réponses sont alors possibles « action associée » et « action non associée ». La situation de chacune des entreprises devrait être reliée à la manière de concevoir le changement. Pour le vérifier, il suffit d'effectuer des tests t pour chacune des caractéristiques du changement selon la situation de l'entreprise à l'égard des seize plans d'action mentionnés.

Un seul des plans d'action ne fait varier aucune des opinions qui ont trait aux caractéristiques du changement. Il s'agit de la mise en place d'un système qualité. Une seule caractéristique du changement n'est associée à aucun des plans d'action: la gestion prévisionnelle des emplois et des compétences. Normalement, entre deux et onze positions sur les aspects du changement subissent l'influence des divers plans. Au total, nous trouvons quatre-vingt-trois tests $t$ significatifs sur un total de 352. Les égalités de moyennes sont donc nettement dominantes, c'est-à-dire que, le plus souvent, il n'y a pas de lien entre le plan d'action et les caractéristiques du changement. Il y a malgré tout un certain nombre de relations significatives. Un plan qui consiste en un développement des exportations ou en la fusion d'entités du même secteur ou d'un secteur complémentaire influe sur onze caractéristiques; s’il se rapporte à l'extension du secteur commercial de l'entreprise, il agit sur dix caractéristiques. Près de $40 \%$ de toutes les relations significatives, donc, correspondent à l'influence de ces trois plans. Nous notons bien, ici, l'incidence des préoccupations commerciales. D'autres plans font aussi varier plusieurs moyennes: une diversification de la production et l'aménagement et la réduction du temps de travail (sept chacun), la mise en conformité avec une norme qualité (six). Les questions de production interviennent fortement elles aussi. Nous constatons à nouveau que les distinctions ont souvent lieu entre les organismes qui sont soumis aux exigences de la commercialisation et ceux qui ne le sont pas. Ces observations se confirment quand nous nous penchons sur les caractéristiques qui se manifestent le plus souvent. L'élévation nécessaire des compétences des salariés et la réorganisation du travail pour augmenter la productivité apparaissent huit fois chacune; la nécessaire diversification des produits, sept fois; l'optimisation de la fonction commerciale et des changements des exigences de la clientèle, six fois chacune; la transformation des techniques de fabrication, cing fois. Une seule caractéristique est constamment absente: le vieillissement de la population des salariés. Toutes les autres connaissent des différences au moins une fois, mais nous voyons bien que si le changement se caractérise par 
la productivité ou par la commercialisation, il différencie davantage. Sur les quatre-vingt-trois inégalités de moyennes, il n'y en a que quatre qui ne montrent pas que la caractéristique du changement est plus importante si le plan mentionné est associé à la stratégie.

\subsection{Perception du changement et constats du développement suite aux formations des salariés}

En principe, la manière dont on conçoit le changement, aujourd'hui, devrait influer sur la façon dont on perçoit le développement de l'entreprise après que la formation des employés ait eu lieu. Or, telle est la situation. Mais dans certains cas seulement. Trois des neuf indices du développement ne sont pas du tout corrélés avec les opinions sur les caractéristiques du changement; il s'agit des capacités d'anticipation, d'autonomie et de communication. Quand c'est bien le cas, il s'agit d'une faible détermination qui est toujours inférieure à $34 \%$. Les sources d'influence sont éparses. Treize caractéristiques interviennent et seulement quatre d'entre elles le font plus d'une fois, soit deux fois: clientèle, système de communication, gestion des emplois et mondialisation. Six des treize corrélations sont négatives; elles impliquent le rapport entre les nouveaux produits et le développement du profil d'emploi des salariés; le système de communication et le développement dans le sens de la polyvalence ou dans le sens des capacités pour intégrer des objectifs de production; la gestion des emplois et des compétences ou les autres entreprises complémentaires et le développement qui a trait aux capacités à intégrer des objectifs de gestion; le vieillissement de la population des salariés et un développement qui consiste, à nouveau, en des capacités pour intégrer des objectifs de production. Ainsi, plus ces caractéristiques sont comprises comme importantes, moins le développement semble appréciable. Les autres corrélations sont positives et elles signalent l'incidence de la fusion, de la clientèle, de la concurrence internationale, de l'élévation des compétences des salariés, de la gestion des emplois et des compétences, de la réorganisation du travail, du plan social, de la mondialisation puis des techniques de fabrication.

\subsection{Perception du changement et contexte dans lequel le projet pour le FSE a été conçu}

Les opinions sur les diverses caractéristiques du changement peuvent dépendre du contexte dans lequel l'entreprise a développé le projet qu'elle a soumis auprès du FSE. Nous pouvons, en effet, penser que ces opinions varieront selon, par exemple, que le chiffre d'affaire était en croissance ou non. Le questionnaire propose cinq indicateurs du contexte auxquelles on répond par oui ou par non. Nous pouvons donc effectuer ici des tests $t$. 


\section{Tableau 5}

Régression multiple de toutes les variables relatives à la teprésentation actuelle du changement $(1=$ de manière incontoumable et $4=$ de manière insignifiante) dont la contribution est apparue significative pour les variables relatives aux constats de développement suite aux formations des salariés $(1=$ très important et $4=$ pas important $)$.

Coefficient standardisé $(\beta)$ et variance expliquée $\left(\mathrm{R}^{2}\right)$

\begin{tabular}{|c|c|c|c|}
\hline $\begin{array}{l}\text { Variables relatives aux constats de développement } \\
\text { suite aux formations des salariés }\end{array}$ & $\begin{array}{l}\text { Variables relatives à la représentation } \\
\text { actuelle du changement }\end{array}$ & $\beta$ & $\mathrm{R}^{2}$ \\
\hline Du profil d'emploi des salariés & $\begin{array}{l}\text { Fusion } \\
\text { Nouveaux produits } \\
\text { Clientèle }\end{array}$ & $\begin{array}{r}0,24 \\
-0,29 \\
0,20\end{array}$ & \\
\hline De la polyvalence & $\begin{array}{l}\text { Système de communication } \\
\text { Concurrence internationale } \\
\text { Élévation des compétences des salariés }\end{array}$ & $\begin{array}{r}-0,38 \\
0,24 \\
0,20\end{array}$ & \\
\hline $\begin{array}{l}\text { Des capacités d'analyse des situations de travail } \\
\text { Des capacités d'anticipation } \\
\text { Des capacités d'autonomie } \\
\text { Des capacités de communication }\end{array}$ & Gestion des cmplois et des compétences & 0 & 0 \\
\hline Des capacités à intégrer des objectufs de gestion & $\begin{array}{l}\text { Gestion des emplois et des compétences } \\
\text { Réotganisarion du travail } \\
\text { Autres entreprises complémentaires } \\
\text { Plan social }\end{array}$ & $\begin{array}{r}-0,24 \\
0,25 \\
-0,24 \\
0,19\end{array}$ & \\
\hline Des capacités pour in régrer des objectifs de production & $\begin{array}{l}\text { Mondialisation } \\
\text { Vieillissement de la population des salariés } \\
\text { lechriques de fabrication } \\
\text { Système de communication } \\
\text { Clientèle }\end{array}$ & $\begin{array}{r}0,29 \\
-0,29 \\
0,26 \\
-0,24 \\
0,20\end{array}$ & \\
\hline Des capacités pour intégrer des exigences qualité & Mondialisation & 0 & \\
\hline
\end{tabular}

Selon, donc, que le chiffre d'affaire était ou non en croissance, trois des vingtdeux opinions sur le changement connaissent une inégalité des moyennes. Ce sont celles qui ont trait aux relations renforcées avec les donneurs d'ordres ${ }^{207}$, à la mise en place d'un système informatique en réseau pour l'ensemble des fonctions de l'entreprise ${ }^{208}$, à la nécessaire diversification des produits $^{209}$ et au vieillissement de la population salariée ${ }^{210}$. Dans les deux premiers cas, les moyennes témoignent de ce que ces aspects importent davantage si l'entreprise ést effectivement en contexte de croissance du chiffre d'affaire; dans les deux autres, c'est le contraire.

Selon que le chiffre d'affaire était stagnant ou non, à nouveau, ce sont quatre caractéristiques dont les différences de moyennes ne peuvent être attribuées au hasard: les relations renforcées avec les donneurs d'ordres ${ }^{211}$, les relations avec

\footnotetext{
$207 \mathrm{t}_{(92,88)}=-2,29 ; \mathrm{p}<0,05$.

${ }^{2018} \mathrm{t}_{(166)}=-2,25 ; \mathrm{p}<0,05$.

${ }^{209} \mathrm{t}_{(165)}=2,28 ; \mathrm{p}<0,05$.

${ }^{210} \mathrm{t}_{(165)}=3,06 ; \mathrm{p}<0,05$.

${ }^{211} \mathrm{t}_{(165)}=3,99 ; \mathrm{p}<0,05$.
} 


\section{NPSS, VOLUME 1, NUMÉRO 1, 2005}

les sous-traitants ${ }^{212}$, la transformation des techniques de fabrication ${ }^{213}$ et la nécessaire diversification des produits ${ }^{214}$. Les trois premières caractéristiques font état d'une moins grande préoccupation si la réponse est affirmative; pour l'autre, c'est l'inverse.

Si le chiffre d'affaire est en baisse, un aspect du changement fait qu'on lui accorde plus d'importance, soit le vieillissement de la population salariée ${ }^{215}$, un autre fait qu'on lui en accorde moins, soit l'aménagement et la réduction du temps de travail ${ }^{216}$.

Ainsi, que l'entreprise connaisse ou non une croissance du chiffre d'affaire, cela ne distingue qu'exceptionnellement les opinions sur le changement. Il semble toutefois que la croissance ou la stagnation coincide avec un surcroit d'intérêt pour les relations avec les donneurs d'ordre ou avec une diminution de cet intérêt pour la diversification des produits; il semble également que la croissance attire un peu plus l'attention vers le problème du vieillissement de la population salariée; il semble encore que la croissance du chiffre d'affaire incite un peu plus à se questionner sur le système informatique.

La croissance peut aussi se rapporter aux effectifs. Si tel est le cas, le changement de direction ${ }^{217}$ ou la concurrence internationale ${ }^{218}$ inquiètent un peu moins, l'aménagement du temps de travail ${ }^{219}$, un peu plus. Si l'on fait l'analyse en fonction de ce que les effectifs soient en baisse, les résultats vont dans le même sens.

\section{Une représentation complexe}

Pour peu qu'on examine l'ensemble des données, il n'y a pas, dans l'esprit des administrateurs, d'évocation de principe absolu, de mise en œuvre de logique linéaire. Comprendre le rôle de la formation dans un organisme, c'est manipuler un ensemble complexe d'informations.

\footnotetext{
$212 \mathrm{t}_{(51,26)}=2,96 ; \mathrm{p}<0,05$.

${ }^{213} \mathrm{t}_{(53,89)}=2,64 ; \mathrm{p}<0,05$.

${ }^{214} \mathrm{t}_{(55,46)}=-2,54 ; \mathrm{p}<0,05$

${ }^{215} \mathrm{t}_{(165)}=-3,20 ; \mathrm{p}<0,05$.

${ }^{216} \mathrm{t}_{(166)}=2,08 ; \mathrm{p}<0,05$.

${ }^{217} \mathrm{t}_{(165)}=2,22 ; \mathrm{p}<0,05$.

${ }^{218} \mathrm{t}_{(125,23)}=2,38 ; \mathrm{p}<0,05$.

${ }^{219} \mathrm{t}_{(169)}=-2,90 ; \mathrm{p}<0,05$.
} 


\section{Figure 1}

Représentation de la contribution du FSE chez les administrateurs

Contexte de la conception de la demande
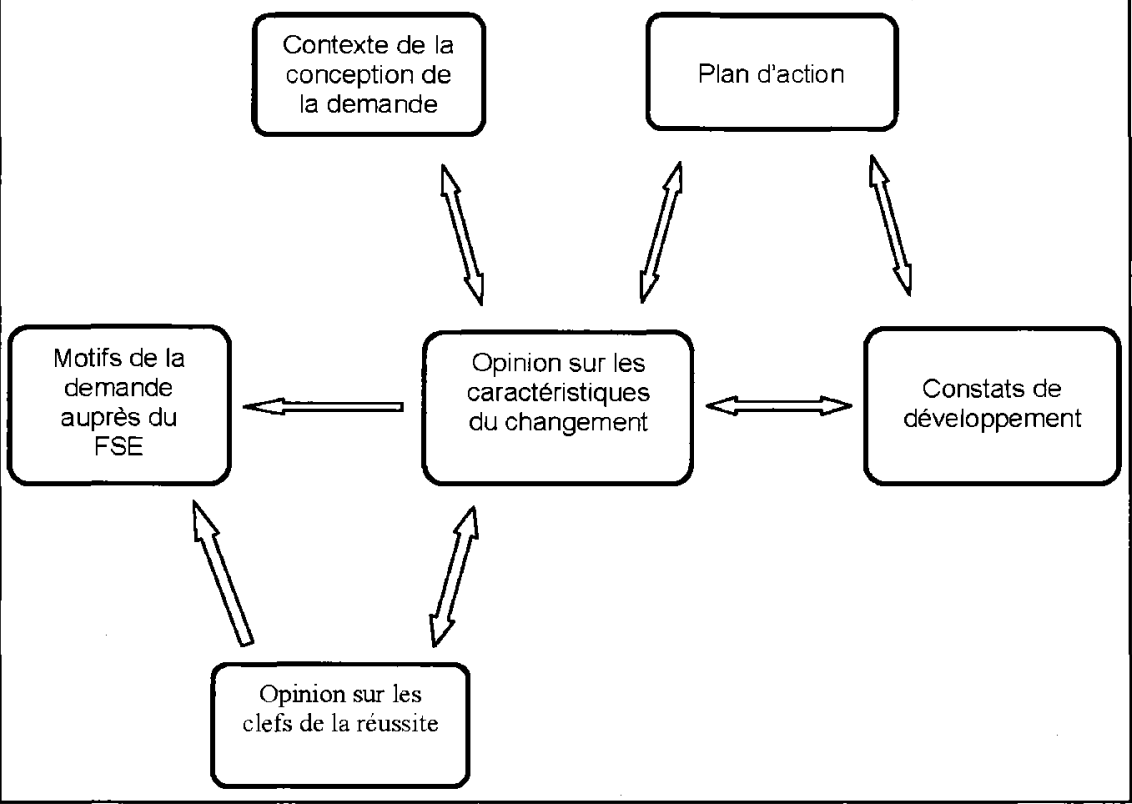

Les raisons pour lesquelles on fait appel à une institution afin qu'elle contribue à la formation des employés de l'organisme dont on est responsable dépend de la manière dont on perçoit l'organisme, de la manière dont on le conçoit en tant qu'il fait partie d'une société en devenir et qu'il constitue lui-même une entité avec ses dynamiques propres. Cette société et cet organisme correspondent à une manière de se représenter, d'une part, le changement et, d'autre part, le contexte dans lequel l'appel à l'institution a lieu, deux représentations qui agissent l'une surl'autre. Le plan d'action qui est envisagé est forcément associé à la conception du changement et il agit ainsi indirectement sur les motifs de la demande. Avoir telle opinion sur le changement, c'est aussi entrevoir des clefs de la réussite et, donc, se donner des motifs pour solliciter une aide à la formation. La manière dont on interprète le développement suite à la formation est définie par l'opinion sur le changement et par le plan d'action; et elle intervient elle-même sur la conception du changement autant que sur le plan d'action en tant que la dynamique est historique, c'est-à-dire qu'elle est toujours en cours. Nous avons bien affaire à une systématisation des informations chez les décideurs, 
à une aptitude continue à adapter l'organisme à l'environnement, à des positions réductibles autant qu'irréductibles. Et il est peu probable que cette manière de percevoir l'entreprise, de percevoir l'entreprise dans le monde ne se soit pas imposée aux observateurs de la DATAR.

\section{Conclusion}

Notre étude avait pour but de répondre à diverses questions, de vérifier diverses hypothèses sur les représentations des responsables des entreprises. Si la problématique était justifiée, l'observation devait repérer une pluralité de perceptions, une aptitude à intervenir sur soi et sur le milieu, une variation des positions et des interventions selon le milieu en même temps que des récurrences attribuables à un environnement plus global, une psyché intriquée aussi bien que nette qui déborde le cadre d'une logique strictement linéaire. Dès les premières analyses, elle a découvert une vision plurielle, nuancée, qui ne se laisse pas réduire à une seule énonciation, qui ne présente pas de positions tranchées. Cette vision se distingue nettement du discours que tient normalement un acteur social, dans les sociétés postmodernes, quand on lui demande de se prononcer sur des questions d'ordre sociopolitique; cet acteur, en effet, prend communément une position dans l'ensemble de celles qui circulent et la tient à l'exclusion des autres, de façon catégorique. Le responsable de l'entreprise, quand il est amené à se prononcer sur l'avenir de cette entreprise, manipule bon nombre d'informations qui le contraignent à un propos multidimensionnel, parfois logique, parfois paradoxal, propos qui, en réalité, correspond à la complexité de la situation. Dans ce propos, les zones d'influence agissent dialectiquement les unes sur les autres: l'environnement externe par rapport aux conditions internes ou encore les contraintes de la mondialisation par rapport à celles de la territorialité. Les analyses subséquentes ont à la fois fait état de variations et de non-variations. Celles qui ont porté sur les moyennes ont révélé des inégalités mais beaucoup plus d'égalités. Celles qui ont cherché des régressions significatives ont trouvé des variables indépendantes qui n'ont pas d'effet, d'autres qui n'en ont que peu; dans les deux cas, elles ont mis en évidence des récurrences. Les égalités de moyennes et la faiblesse des variances expliquées rappellent que les sociétés postindustrielles évoluent vers l'indifférenciation. Les inégalités de moyennes soulignent que tout n'est pas qu'indifférenciation; les variances expliquées confirment que des éléments agissent les uns sur les autres, que les significations sont portées partiellement par d'autres significations dans un univers de représentations. La récurrence de certaines déterminations et de certaines 
indéterminations démontre que la postmodernité produit des ensembles qui ouvrent la socialité sur la similitude autant que sur la dissimilitude. C'est dans ce contexte de probabilités, ouvert mais non infini, que les responsables des entreprises savent recourir, de manière particulière et commune, à une institution commune pour orienter leur entreprise de façon particulière et semblable.

\section{Références}

Alcaras, Jean-Robert (1997). Sur la modélisation des processus d'adaptation socio-économiques: aspects symboliques et téléologiques de l'autonomie individuelle et sociale; interprétation des stratégies économiques en termes d'ingénierie. Thèse de Doctorat en sciences économiques soutenue le 10 janvier 1997, Université d'Aix-Marseille, Faculté d'économie appliquée, Groupe de Recherche sur l'Adaptation, la Systémique et la Complexité Économique (U.R.A., C.N.R.S. Numéro 935).

Alcaras, Jean-Robert et Lacroux, François (2001). «Planifier c'est s'adapter ». Publication du site Internet Association pour la modélisation de la Complexité consultée en juillet 2004, http://www.mcxapc.org/docs/ateliers/padapte.pdf.

Balandier, Georges (2001). Le Grand systeme. Paris, Fayard.

Bagaoui, Rachid (1999). Culture d'entreprise te compromis patronal-syndical. Sudbury, Série monographique en sciences humaines.

Boyer, Robert et Freyssenet, Michel (1995). Émergence de nouveaux modeles industriels; problématique et démarche d'analyse. Actes du GERPISA, n'15.

Castels, Manuel (1996). The Rise of the Network Society. Volume I, The Information Age: Economy, Society and Culture. Oxford, Blackwell.

Cogburn, Dennis L. (1998). "Globalization and State Autonomy in the Information Age: Telecommunications Sector Restructuring in South A frica ". Journal of International Affairs, 51 (2), printemps, 583-604.

Commission Européenne (1995). Orientations communes pour le suivi et les évaluations intermédiaires. Office des publications officielles des communautés européennes.

Crozier, Michel et Friedberg, Erhard (1977). L'acteur et le système. Paris, Seuil, Coll. Points.

Darwin, Charles (1983). L'Origine des espèces au moyen de la sélection naturelle, ou la lutte pour l'existence dans la nature. Paris, Maspéro, [1859].

Held, David et McGrew, Anthony (2002). Globalization/ Antiglobalization. Cambridge, Polity Press.

Hofstede, Geert (1984). Culture's Consequences: International Differences in Work-Related V alues. Beverly Hills, Sage.

Jacot, J.H. (dir.) (1990). Du Fordisme au toyotisme? Les voies de la modernisation du système antomobile en France et au Japon. Paris, La Documentation française.

Jaquet, Nicolas, Délégation à l'Aménagement du Territoire et à l'Action Régionale (DATAR) (2004). La France, puissance industrielle. Février 2004.

Laflamme, Simon (1992). La Société intégrée. De la circulation des biens, des idées et des personnes. New York, Paris, Peter Lang. 
Laflamme, Simon (1995). Communication et émotion. Essai de microsociologie relationnelle. Paris, L'Harmattan, Coll. Logiques sociales.

Laflamme, Simon et Reguigui, Ali (2003). Homogénéité et distinction. Sudbury, Prise de parole, Coll. Ancrages.

Lamarck, Jean-Baptiste (1968). La Pbilosopbie zoologique. Paris, Union générale d'éditions, [1873].

Le Moigne, Jean-I_ouis (1995). La Modélisation des systèmes complexes. Paris, Dunod.

Luhmann, Niklas (1995). Social Systems. Stanford (CA), Stanford University Press.

Ministère de l'emploi et de la solidarité (1994), DOCUP Objectif IV (1994). Favoriser l'adaptation des travailleurs aux mutations industrielles et à l'évolution des systèmes de production.

McPhail, Thomas I. (2001). Global Communication. Theories, Stakebolders, and Trends. Boston, Allyn \& Bacon.

Mowlana, Hamid (1996). Global Communication In Transition: The End of Diversity?, Thousand Oaks (CA), Sage.

Morin, Edgar (1977-2003). La Méthode. Tomes I-VI. Paris, Seuil.

Morin, Edgar et Le Moigne, Jean-Louis (1999). L'Intelligence de la complexité, Paris, L'Harmattan.

Nelson, Richard R. et Winter, Sidney G. (1982). An Evolutionary Theory of Economic Change. Harvard University Press.

Simon, Herbert A. (1983). Administration et processus de décision. Paris, Economica.

Simon, Herbert A. (1991). Science des systèmes, science de l'artificiel. Paris, Dunod.

Taché, Alain (2003). L'Adaptation: un concept sociologique systémique. Paris, L'Harmattan.

Tort, Patrick (1996). Dictionnaire du darwinisme et de l'évolution. Trois volumes. Paris, PUF.

Williamson, Oliver E. (1974). « Exit and Voice : Some Implications for the Study of the Modern Corporation $»$. Social Science Information, 13 (6), décembre, 61-72.

Williamson, Oliver E. (1975). Markets and Hierarchies. Analysis and antitrust implications. Free Press, Macmillan.

Williamson, Oliver E. (1976). "The Economics of Internal Organization : Exit and voice in relation to market and hierarchies \%. American Economic Review, papers and proceedings, 66 (2), mai, 369-377.

Zarifian, Philippe et Palloix, Christian (1988). La société post-économique. Paris, L'Harmattan. 